\title{
Investigating the Chemolithoautotrophic and Formate Metabolism of Nitrospira moscoviensis by Constraint-Based Metabolic Modeling and ${ }^{13} \mathrm{C}$-Tracer Analysis
}

\author{
(DChristopher E. Lawson, a,d Aniela B. Mundinger, ${ }^{b}$ (D) Hanna Koch, ${ }^{b}$ Tyler B. Jacobson, ${ }^{\text {d }}$ Coty A. Weathersby, (D) Mike S. M. Jetten, \\ (D) Martin Pabst, c (D)Daniel Amador-Noguez, ${ }^{\mathrm{a}, \mathrm{b}}$ (D) Daniel R. Noguera, ${ }^{\mathrm{a}, \mathrm{d}}$ (D) Katherine McMahon, ${ }^{\mathrm{a}, \mathrm{d}, \mathrm{e}}$ (D) Sebastian Lücker ${ }^{\mathrm{b}}$

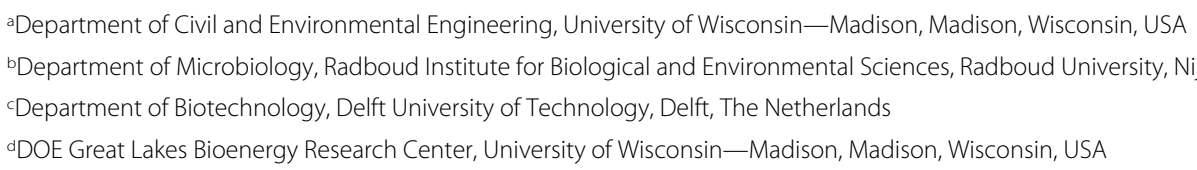

Christopher E. Lawson and Aniela B. Mundinger contributed equally to this work. Author order was determined based on contributions to writing

ABSTRACT Nitrite-oxidizing bacteria belonging to the genus Nitrospira mediate a key step in nitrification and play important roles in the biogeochemical nitrogen cycle and wastewater treatment. While these organisms have recently been shown to exhibit metabolic flexibility beyond their chemolithoautotrophic lifestyle, including the use of simple organic compounds to fuel their energy metabolism, the metabolic networks controlling their autotrophic and mixotrophic growth remain poorly understood. Here, we reconstructed a genome-scale metabolic model for Nitrospira moscoviensis (iNmo686) and used flux balance analysis to evaluate the metabolic networks controlling autotrophic and formatotrophic growth on nitrite and formate, respectively. Subsequently, proteomic analysis and $\left[{ }^{13} \mathrm{C}\right]$ bicarbonate and $\left[{ }^{13} \mathrm{C}\right]$ formate tracer experiments coupled to metabolomic analysis were performed to experimentally validate model predictions. Our findings corroborate that $N$. moscoviensis uses the reductive tricarboxylic acid cycle for $\mathrm{CO}_{2}$ fixation, and we also show that $N$. moscoviensis can indirectly use formate as a carbon source by oxidizing it first to $\mathrm{CO}_{2}$ followed by reassimilation, rather than direct incorporation via the reductive glycine pathway. Our study offers the first measurements of Nitrospira's in vivo central carbon metabolism and provides a quantitative tool that can be used for understanding and predicting their metabolic processes.

IMPORTANCE Nitrospira spp. are globally abundant nitrifying bacteria in soil and aquatic ecosystems and in wastewater treatment plants, where they control the oxidation of nitrite to nitrate. Despite their critical contribution to nitrogen cycling across diverse environments, detailed understanding of their metabolic network and prediction of their function under different environmental conditions remains a major challenge. Here, we provide the first constraint-based metabolic model of Nitrospira moscoviensis representing the ubiquitous Nitrospira lineage II and subsequently validate this model using proteomics and ${ }^{13} \mathrm{C}$-tracers combined with intracellular metabolomic analysis. The resulting genome-scale model will serve as a knowledge base of Nitrospira metabolism and lays the foundation for quantitative systems biology studies of these globally important nitrite-oxidizing bacteria.

KEYWORDS lithoautotrophic metabolism, metabolic modeling, metabolomics, proteomics, systems biology
Citation Lawson CE, Mundinger AB, Koch H Jacobson TB, Weathersby CA, Jetten MSM, Pabst M, Amador-Noguez D, Noguera DR, McMahon K, Lücker S. 2021. Investigating the chemolithoautotrophic and formate metabolism of Nitrospira moscoviensis by constraint-based metabolic modeling and ${ }^{13} \mathrm{C}$ tracer analysis. mSystems 6:e0173-21. https:// doi.org/10.1128/mSystems.00173-21.

Editor Steven J. Hallam, University of British Columbia

Ad Hoc Peer Reviewer (D) Brett L. Mellbye, Oregon State University

Copyright $\odot 2021$ Lawson et al. This is an open-access article distributed under the terms of the Creative Commons Attribution 4.0 International license.

Address correspondence to Christopher E. Lawson, c.e.lawson.87@gmail.com, or Sebastian Lücker, s.luecker@science.ru.nl.

Received 16 February 2021

Accepted 21 July 2021

Published 17 August 2021 
- he oxidation of nitrite to nitrate is a key step in nitrification and the global nitrogen cycle. The process is a critical control point counteracting nitrogen loss to the atmosphere and is mediated by a phylogenetically diverse functional guild known as the nitrite-oxidizing bacteria (NOB) (1). The genus Nitrospira constitutes the most diverse and abundant NOB based on marker gene (16S rRNA and nitrite oxidoreductase [NXR]) and metagenomic surveys. The genus Nitrospira consists of at least six lineages that mediate nitrite oxidation across various habitats, including soil, freshwater, marine, terrestrial, and engineered ecosystems $(1,2)$. Nitrospira must be flexible enough to survive in the wide range of fluctuating environmental conditions characteristic of these habitats, suggesting its ecophysiology and ecological niches extend beyond those initially defined by its chemolithoautotrophic lifestyle.

Despite their recalcitrance to cultivation, recent insights driven by metagenomics have shed light on the unique features of Nitrospira's carbon and energy metabolism (3). Nitrospira harbors novel respiratory chain enzymes for energy conservation, including an evolutionarily distinct membrane-bound periplasmic NXR and a putative cytochrome $b d$-like oxidase that may allow it to adapt to low-dissolved-oxygen environments (3). Nitrospira also harbors all genes for $\mathrm{CO}_{2}$ fixation via the reductive tricarboxylic acid cycle (rTCA) and lacks the two key genes (for ribulose bisphosphate carboxylase and phosphoribulokinase) needed to operate the Calvin-Benson-Bassham cycle (CBB) used by some NOB (3). Outside their chemolithoautotrophic growth, genomic and experimental data have revealed that Nitrospira spp. can use alternative substrates to fuel their carbon and energy metabolism $(1,4,5)$. In addition to nitrite, some Nitrospira species have been experimentally shown to use formate, hydrogen, and ammonia as electron donors with oxygen or nitrate as terminal electron acceptors (4-9). For example, Nitrospira moscoviensis contains a soluble formate dehydrogenase and NiFe hydrogenase that allow growth with formate and $\mathrm{H}_{2}$, respectively $(4,5)$, whereas the recently discovered complete ammonia-oxidizing (comammox) Nitrospira contains pathways for both ammonia and nitrite oxidation that enable growth via complete nitrification $(7,8)$. In addition to their energy metabolism, fluorescence in situ hybridization combined with microautoradiography (FISH-MAR) experiments have also suggested that Nitrospira populations present within activated sludge microbial communities can assimilate pyruvate (2) and formate (10), although the carbon assimilation pathways for these substrates have yet to be determined. While this expanded metabolic versatility suggests that Nitrospira are adapted to dynamic environmental conditions, our ability to predict their function in natural and engineered ecosystems is constrained by the limited understanding of their metabolic network and the lack of quantitative tools to study their metabolism.

Genome-scale metabolic modeling is a powerful method for analyzing and predicting the biochemical pathways driving microbial metabolism. Such modeling approaches calculate the flow of metabolites through a reconstructed metabolic network based on relevant constraints (e.g., network stoichiometry, thermodynamics, and measured fluxes) using a technique called flux balance analysis (FBA) (11). This provides a quantitative framework for analyzing metabolism and predicting phenotypes when combined with physiological data. Moreover, genome-scale models can be used to generate testable hypotheses on the functional capabilities of organisms under defined conditions that can subsequently be tested, for instance using ${ }^{13} \mathrm{C}$ isotope tracing combined with metabolomics (12).

Here, we provide the first constraint-based metabolic reconstruction and analysis of $N$. moscoviensis representing the ubiquitous Nitrospira lineage II. We examine the metabolism of $N$. moscoviensis growing under chemolithoautotrophic conditions and also during growth with formate as a substrate. Taking advantage of recent advances to cultivate Nitrospira in continuous flow membrane bioreactors (13), we subsequently validate $N$. moscoviensis' predicted metabolic network using proteomics and ${ }^{13} \mathrm{C}$ tracers combined with quantitative metabolomic analysis. Our proteomic and ${ }^{13} \mathrm{C}$ metabolomic results corroborate the use of the rTCA for carbon fixation by $N$. moscoviensis during chemolithoautotrophic growth. We further show that $N$. moscoviensis does not 


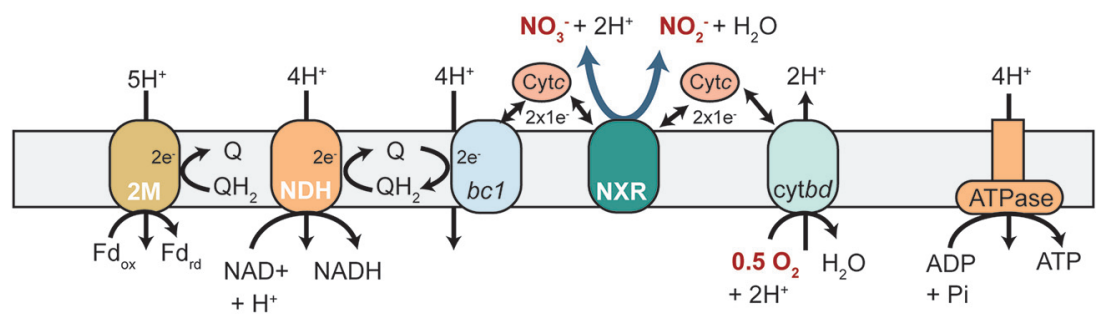

Cytoplasm

FIG 1 Theoretical model for the respiratory chain of Nitrospira moscoviensis as hypothesized in this study. $\mathrm{Q}$, quinone; $\mathrm{QH}_{2}$, quinol; $\mathrm{Fd}$, ferredoxin; Cytc, cytochrome $c ; 2 \mathrm{M}, 2 \mathrm{M}$-type complex I; $\mathrm{NDH}$, NADH dehydrogenase (complex I); $b c 1$, cytochrome $b c_{1}$ complex (complex III); NXR, nitrite oxidoreductase; cytbd, cytochrome $c$ oxidase (complex IV).

assimilate formate directly, but instead reassimilates $\mathrm{CO}_{2}$ produced via formate oxidation using the rTCA cycle. The resulting genome-scale model (GEM), iNmo686, will serve as a knowledge base for understanding and predicting the function of Nitrospira in both natural and engineered ecosystems.

(This article was submitted to an online preprint archive [14].)

\section{RESULTS}

Genome-scale metabolic reconstruction of Nitrospira moscoviensis. The genomescale metabolic network of $\mathrm{N}$. moscoviensis (iNmo686) was reconstructed from the most recent $N$. moscoviensis genome annotation (NCBI accession number NZ_CP011801), aided by reaction annotations in the MetaCyc (15) and ModelSEED databases available through the Department of Energy systems biology knowledgebase (16). Proteomic analysis of $N$. moscoviensis grown on nitrite was also performed to confirm that model reactions with nonzero flux values were detected in the proteome (see Table S1 in the supplemental material). The final reconstruction contained a total of 678 reactions, 638 metabolites, and 686 genes (see Data Set S1). Reactions for Nitrospira's respiratory chain were reconstructed based on existing models of electron flow $(1,3)$ and assuming that the two protons produced during nitrite oxidation in the periplasm contribute to proton motive force generation. Cytoplasmic reactions for formate dehydrogenase and hydrogenase that catalyze formate oxidation to $\mathrm{CO}_{2}$ and hydrogen oxidation, respectively, were also included based on genomic and experimental evidence $(4,5)$. Formate transport and assimilation reactions mediated by a putative reductive glycine pathway (17) encoded in the genome (Data Set S1) were also included to evaluate the possibility of directly assimilating formate as a carbon source, as suggested by previous FISH-MAR observations (10).

Since the stoichiometry for proton translocation in Nitrospira's respiratory chain complexes is unknown, values were assumed from model organisms (Data Set S1; Fig. 1). The mechanism for reducing low-potential electron carriers, such as ferredoxin, required for carbon fixation in Nitrospira is also unknown, but it has been hypothesized that the 2M-type NADH dehydrogenase complex (complex I) in Nitrospira performs this function (18). Thus, the model included this mechanism for ferredoxin reduction. All annotated biosynthetic and biodegradation reactions for amino acids, nucleic acids, carbohydrates, lipids, and cofactors were included in the reconstruction (see Data Sets 1 and 2). This encompassed all predicted central carbon metabolic reactions annotated in the genome, including reactions of the rTCA cycle, gluconeogenesis, the pentose phosphate pathway, anaplerotic reactions, one-carbon metabolism, and fatty acid metabolism. Gaps in the network were identified and filled manually to ensure that the model could grow on minimal NOB medium (see Materials and Methods).

To obtain qualitative and quantitative outputs from a genome-scale model via FBA, an objective function is required. This is typically accomplished based on a biomass objective function, which assumes that maximization of biomass growth rate is the cellular objective 
TABLE 1 N. moscoviensis biomass composition

\begin{tabular}{lll}
\hline & \% dry wt & \\
\cline { 2 - 3 } Molecule & Avg & SD \\
\hline RNA & 2.4 & 0.04 \\
DNA $^{a}$ & 1 & ND $^{b}$ \\
Proteins & 46.7 & 4.7 \\
Carbohydrates & 26.9 & 1.8 \\
Lipids & 16.3 & 3.2 \\
Inorganics $^{a}$ & 5 & ND \\
\hline
\end{tabular}

avalues for DNA and inorganics were assumed based on values from Neidhardt et al. (53).

${ }^{b} \mathrm{ND}$, not determined.

(19). Therefore, to generate a representative biomass objective function for N. moscoviensis, we experimentally determined its biomass composition (i.e., macromolecular components) during chemolithoautotrophic steady-state growth on nitrite (see Materials and Methods).

A summary of the biomass composition for N. moscoviensis is presented in Tables 1 and 2. These measurements, together with the genome sequence data and published fatty acid composition data (20), were used to formulate $N$. moscoviensis' biomass objective function (Data Set S1). Growth- and nongrowth-associated maintenance energy requirements were estimated to be $535 \mathrm{mmol}$ ATP $\mathrm{g}$ dry weight ${ }^{-1}$ and $0.90 \mathrm{mmol}$ ATP g dry weight ${ }^{-1} \mathrm{~h}^{-1}$, respectively, by plotting the experimentally determined nitrite uptake rate against the growth rate and using a net ATP yield of $1.0 \mathrm{mmol}$ ATP $\mathrm{mmol} \mathrm{NO}_{2}{ }^{-}-\mathrm{N}^{-1}$ determined from the model (see Fig. S1).

Proteome of $\mathbf{N}$. moscoviensis grown on nitrite. A high-coverage proteome of $N$. moscoviensis grown on nitrite was obtained to confirm expression of the reconstructed metabolic network. Enzymatic digests using three different digestion enzymes alone and in combinations were tested to ensure a representative membrane proteome was obtained (see Text S1 for details). Whole-cell proteome analysis resulted in the detection of 2,519 of the 4,733 nonidentical proteins (53.2\%) encoded in the $N$. moscoviensis genome, including 344 of the 878 nonidentical membrane proteins (39.2\%) (Fig. 2; Table S1). The resulting proteome confirmed expression of genes involved in $N$. moscoviensis carbon and energy metabolism represented in the model, including the nitrite oxidation system (NXR), the reductive TCA cycle, and nitrogen assimilation pathways (see Table S2).

The measured proteome qualitatively corresponded to the recently published transcriptome of $N$. moscoviensis (13), where $86 \%$ of all detected proteins also displayed gene expression levels above the median level (Fig. 2; Table S1). However, protein and

TABLE $2 \mathrm{~N}$. moscoviensis amino acid composition

\begin{tabular}{lll}
\hline & Mass $(\boldsymbol{\mu m o l} / \mathbf{m g}$ dry $\mathbf{w t})$ & \\
\cline { 2 - 3 } Amino acid & Value & SD \\
\hline Ala & 300.6 & 45.7 \\
Arg & 311.8 & 63.9 \\
Asp & 82.3 & 12.3 \\
Glu & 85 & 16.1 \\
Gly & 825.2 & 116.1 \\
His & 37.7 & 2.6 \\
lle & 198.7 & 62.1 \\
Leu & 309.2 & 66.6 \\
Lys & 105.9 & 22.2 \\
Met & 194 & 22.8 \\
Phe & 224.3 & 107.9 \\
Pro & 641 & 227 \\
Ser & 297.5 & 62.6 \\
Thr & 277.2 & 70 \\
Tyr & 41.9 & ND \\
Val & 374 & 59.2 \\
\hline
\end{tabular}

${ }^{a} \mathrm{ND}$, not determined. 

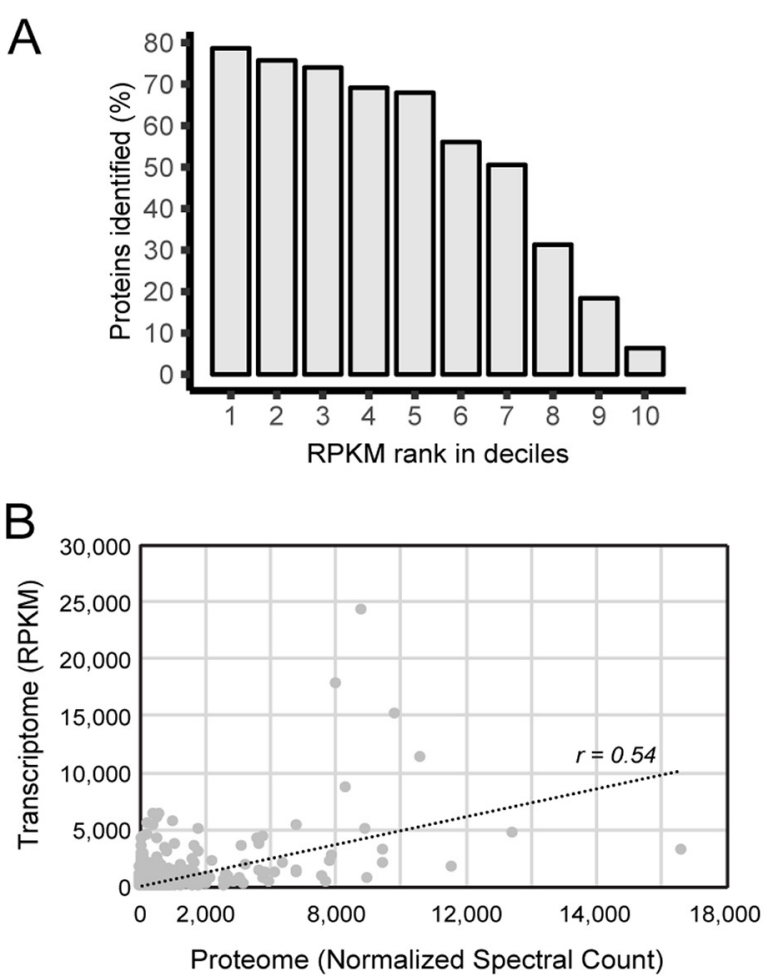

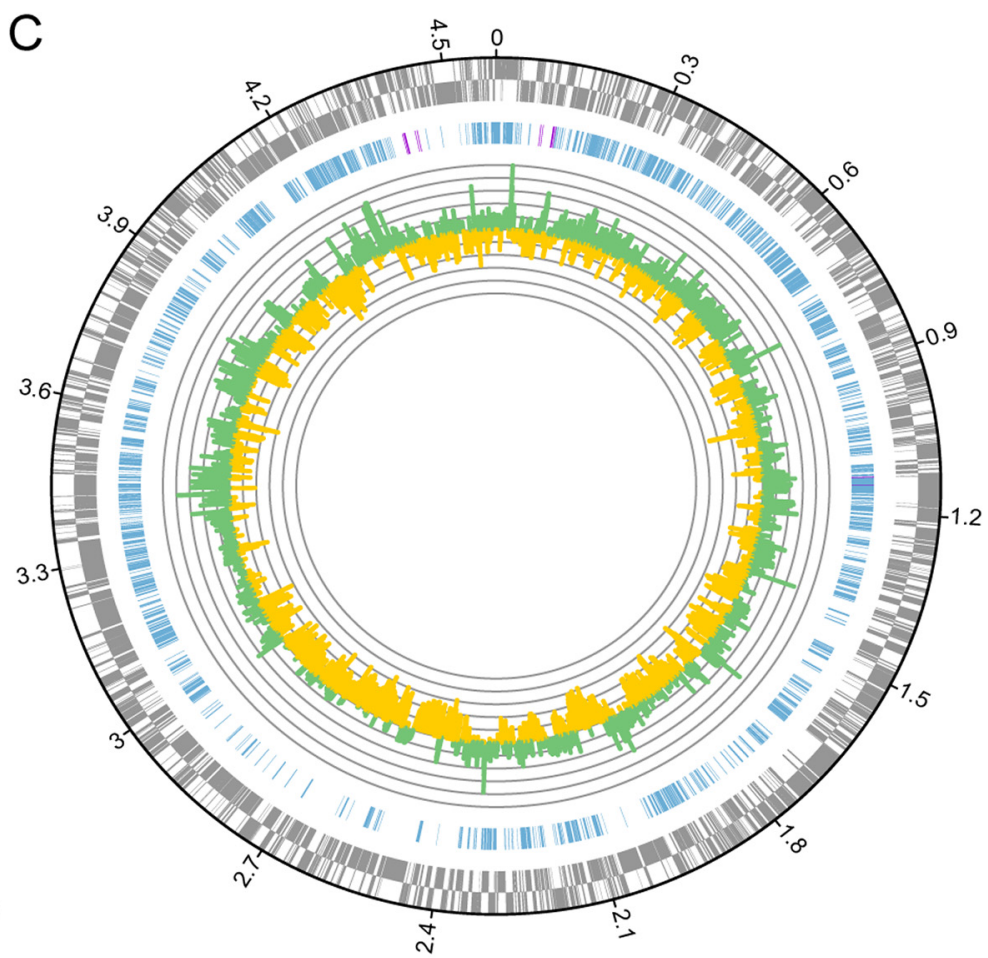

FIG 2 Nitrospira moscoviensis proteomic analysis. (A) Numbers of proteins (given per unique UniProt identifier) identified in the whole-cell proteome compared to their gene expression level, ranked by RPKM in deciles from high (1) to low (10). (B) Pearson correlation ( $r=0.54, n=2,788)$ between open reading frame (ORF) transcript abundance (RPKM) and protein abundance (normalized spectral counts) across all ORFs with detected peptides (see Table S1 in the supplemental material). (C) Genome-wide proteomic and transcriptomic profile of $N$. moscoviensis during growth on nitrite. Rings from outside to inside: (i) ORFs ( \pm strand) predicted in the published $N$. moscoviensis genome (CP011801); (ii) CDSs corresponding to proteins identified in the whole-cell proteome, with $100 \%$ identical CDSs marked in purple; (iii) average gene expression levels ( $n=3$, with 3 technical replicates each) in log, fold to median, with green representing transcription levels above and yellow representing those below the median, shown in log ${ }_{2}$ values.

transcript abundances were only moderately correlated when quantitatively compared using normalized spectral counts as a proxy for protein abundance ( $r=0.54)$ (Fig. 2B). While some of the highly transcribed open reading frames (ORFs) that lacked detection on the proteome level represent nonprotein coding sequences (such as NITMOv2_0031 and NITMOv2_0852), there were also proteins which, despite relatively high transcript levels, were not detected at all in the proteome, such as ATP-dependent RNA helicase RhIE (NITMOv2_1402) and the periplasmic phosphate-binding protein (PstS) of the phosphate ABC transporter (NITMOv2_4757). Such discrepancies can be caused by posttranscriptional regulation or limitations of the proteomic method, such as insufficient solubilization or the presence of few potential cleavage sides for the tryptic digest.

Analysis of chemolithoautotrophic growth on nitrite. The iNmo686 genome-scale model was first used to quantify the carbon and electron flux distribution in N. moscoviensis during aerobic growth on mineral medium with nitrite as an electron donor and $\mathrm{CO}_{2}$ as a carbon source. The model was constrained by the nitrite uptake rate measured during chemostat cultivation, and the objective function was maximizing growth. Consistent with experimental data, when the nitrite uptake rate was set to $8.5 \mathrm{mmol}$ $\mathrm{NO}_{2}^{-} \mathrm{g}_{\text {dry }}$ weight ${ }^{-1} \mathrm{~h}^{-1}$, the specific biomass growth rate equaled $0.006 \mathrm{~h}^{-1}(\sim 116-\mathrm{h}$ doubling time) (Fig. S1). Under these conditions, approximately $66 \%$ of the oxidized $\mathrm{NO}_{2}{ }^{-}$is used for growth-associated maintenance (GAM; polymerization of amino acids into proteins, RNA error checking, etc.) and $17 \%$ is oxidized for nongrowth-associated maintenance (NGAM; maintenance of chemical gradients, turgor pressure, etc.) in the form of ATP (see Fig. S2). The remaining $17 \%$ is used to generate ATP (7\%) and reducing equivalents (10\%) required for $\mathrm{CO}_{2}$ fixation and synthesis of macromolecular building blocks (e.g., central metabolites, amino acids, nucleotides, and fatty acids). Electron transport chain (ETC) reactions involved in nitrite oxidation carried the highest flux and 


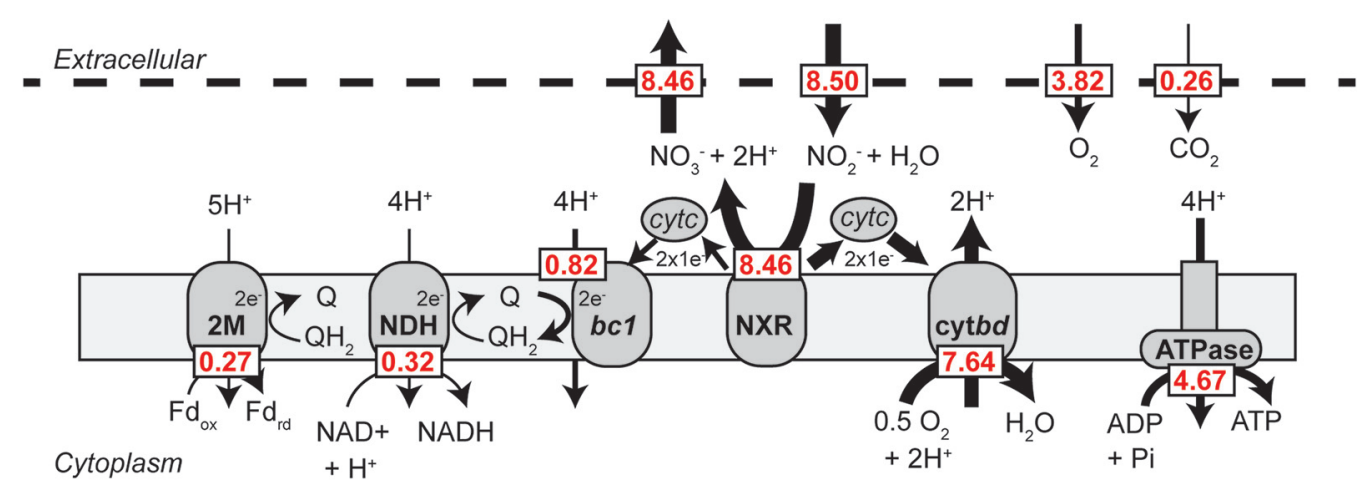

FIG 3 Electron flux distribution predicted via flux balance analysis during chemolithotrophic growth on nitrite. The model was constrained to a nitrite uptake rate of $8.5 \mathrm{mmol} \mathrm{g}$ dry weight ${ }^{-1} \mathrm{~h}^{-1}$ and the biomass growth rate was $0.006 \mathrm{~h}^{-1}$. Numerical values (red) are calculated fluxes in units of millimoles per gram dry weight per hour. Model reactions, compounds, and FBA solutions can be found in Data Set S1. Abbreviations are identical to those in Fig. 1.

were at an order of magnitude higher than carbon fixation fluxes, where the predicted amount of carbon fixed per mole of nitrite oxidized was $32 \mathrm{~mol} \mathrm{C/mol} \mathrm{N} \mathrm{(Fig.} 3$ and 4). This agrees with $N$. moscoviensis' large maintenance energy demand and the high abundance of ETC proteins measured in the proteome (Table S2).

The predicted carbon flux distribution in N. moscoviensis during chemolithoautotrophic growth (Fig. 4) showed that enzymes of the rTCA cycle, including 2-oxoglutarate:ferredoxin oxidoreductase (OFOR), succinyl coenzyme A synthetase (SCS), ATP citrate lyase, $(A C L)$, and pyruvate:ferredoxin oxidoreductase (PFOR), carried the highest carbon flux, consistent with their primary role in $\mathrm{CO}_{2}$ fixation. Pyruvate carboxylase also carried high carbon flux, which is required to replenish TCA cycle intermediates used as precursors for biosynthesis (e.g., oxaloacetate). These flux values agree with the high protein expression levels of rTCA cycle enzymes measured in the N. moscoviensis proteome (Table S2) and gene expression recently reported in the transcriptome (13). In particular, isocitrate dehydrogenase, 2-oxoglutarate:ferredoxin oxidoreductase, and pyruvate:ferredoxin oxidoreductase were the most abundant TCA cycle enzymes (Table S2). This is consistent with the large thermodynamic barriers encountered by the consecutive reactions of alpha-ketoglutarate synthase and isocitrate dehydrogenase $(\Delta G>40 \mathrm{~kJ} / \mathrm{mol})$, as well as pyruvate: ferredoxin oxidoreductase $(\Delta G>14 \mathrm{~kJ} / \mathrm{mol})(21)$.

Generation of reducing equivalents for carbon fixation in $N$. moscoviensis is predicted to result from reverse electron flow from the cytochrome $c$ pool (3). Overall, the model predicts that approximately $3 \%\left(0.23 \mathrm{mmol} \mathrm{g}\right.$ dry weight $\left.\mathrm{t}^{-1} \mathrm{~h}^{-1}\right)$ of the oxidized nitrite is used to generate quinol (largely for nitrite assimilation via octaheme nitrite reductase and reduction of fumarate via succinate dehydrogenase), $4 \%$ ( $0.32 \mathrm{mmol} \mathrm{g}$ dry weight $\left.^{-1} \mathrm{~h}^{-1}\right)$ is used to generate NADH (via complex I), and $3 \%(0.27 \mathrm{mmol} \mathrm{g}$ dry weight $^{-1} \mathrm{~h}^{-1}$ ) is used to generate reduced ferredoxin (via the 2M-type complex I) (Fig. S2). Reduction of $\mathrm{NADP}^{+}$to $\mathrm{NADPH}$, the main electron carrier for biosynthetic reactions, was predicted to occur through $\mathrm{NAD}(\mathrm{P})^{+}$transhydrogenase. While $\mathrm{N}$. moscoviensis harobrs three $\mathrm{NAD}(\mathrm{P})^{+}$transhydrogenases, only NITMOv2_1092 was detected in the proteome (Table S1), indicating that this is the main transhydrogenase during chemolithoautotrophic growth.

Confirmation of autotrophic metabolism with $\left[{ }^{13} \mathrm{C}\right]$ bicarbonate tracer experiments. To confirm the biosynthetic pathways predicted by genome-scale modeling, isotopic tracers combined with high-resolution metabolomics was used to follow ${ }^{13} \mathrm{C}$-labeled bicarbonate incorporation into the metabolome of $N$. moscoviensis. Cells were grown in a membrane bioreactor under steady-state conditions, and $\left[{ }^{13} \mathrm{C}\right]$ bicarbonate was rapidly introduced into the bioreactor, which equilibrated with the dissolved inorganic carbon (DIC) pool in the liquid medium to approximately $65 \%{ }^{13} \mathrm{C}$ enrichment. Following $\left[{ }^{13} \mathrm{C}\right]$ bicarbonate addition, multiple metabolite samples were collected from the bioreactor over a 2-h time period by rapid quenching and 


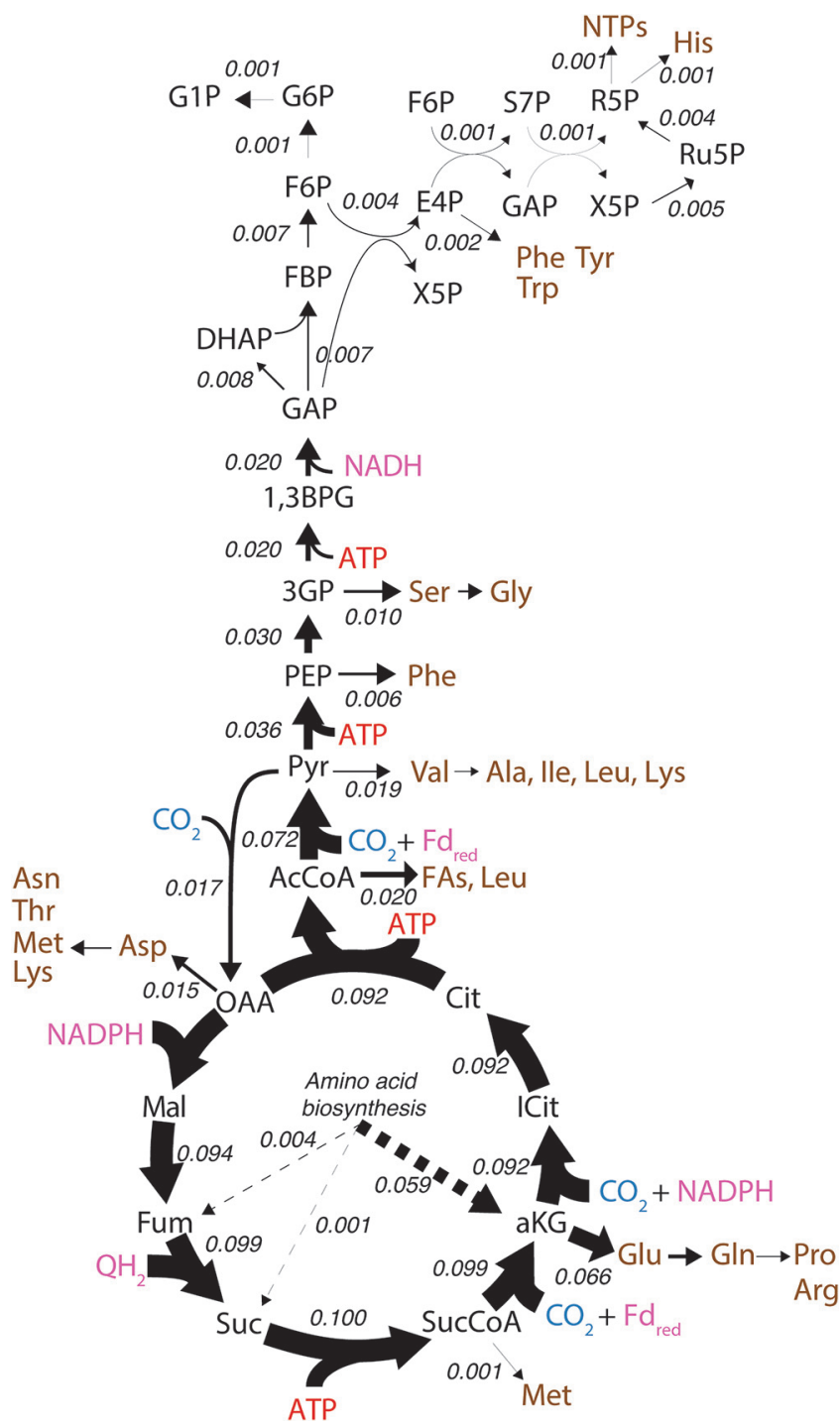

FIG 4 Carbon flux distribution predicted via flux balance analysis during chemolithoautotrophic growth on nitrite. The model was constrained to a nitrite uptake rate of $8.5 \mathrm{mmol} \mathrm{g}$ dry weight ${ }^{-1} \mathrm{~h}^{-1}$ and the biomass growth rate was $0.006 \mathrm{~h}^{-1} \cdot \mathrm{CO}_{2}$ is shown in blue, amino acids and other biomass precursors are shown in brown, ATP is shown in red, and reducing equivalents are shown in pink. Numerical values are calculated fluxes in units of millimoles per gram dry weight per hour. Model reactions, compounds, and FBA solutions can be found in Data Set S1.

extraction of N. moscoviensis cells. A time zero sample corresponding to the period immediately before $\left[{ }^{13} \mathrm{C}\right]$ bicarbonate addition was also collected as a control.

Consistent with the operation of the rTCA cycle, high ${ }^{13} \mathrm{C}$ label incorporation was measured in phosphoenolpyruvate (PEP), acetyl coenzyme A (acetyl-CoA), succinate, and aspartic acid (used as oxaloacetate surrogate) (Fig. 5). Other TCA cycle metabolites showed considerable ${ }^{13} \mathrm{C}$ enrichment, including malate, fumarate, and alpha-ketoglutarate, although to a lower extent than for PEP, acetyl-CoA, succinate, and aspartic acid (Fig. 5). This suggested that the ${ }^{13} \mathrm{C}$ enrichment of malate, fumarate, and alpha-ketoglutarate may have been diluted by inactive pools of these metabolites that were not labeled. Further evidence for this was observed from the faster labeling of glutamate and glutamine than that of alpha-ketoglutarate, which was unexpected because these amino acids are derived from alpha-ketoglutarate (Fig. 5; Data Set S2). We hypothesize that these patterns may reflect substrate channeling in the rTCA cycle of $N$. moscoviensis, which has previously been observed in the TCA cycle of other organisms (22-24). 

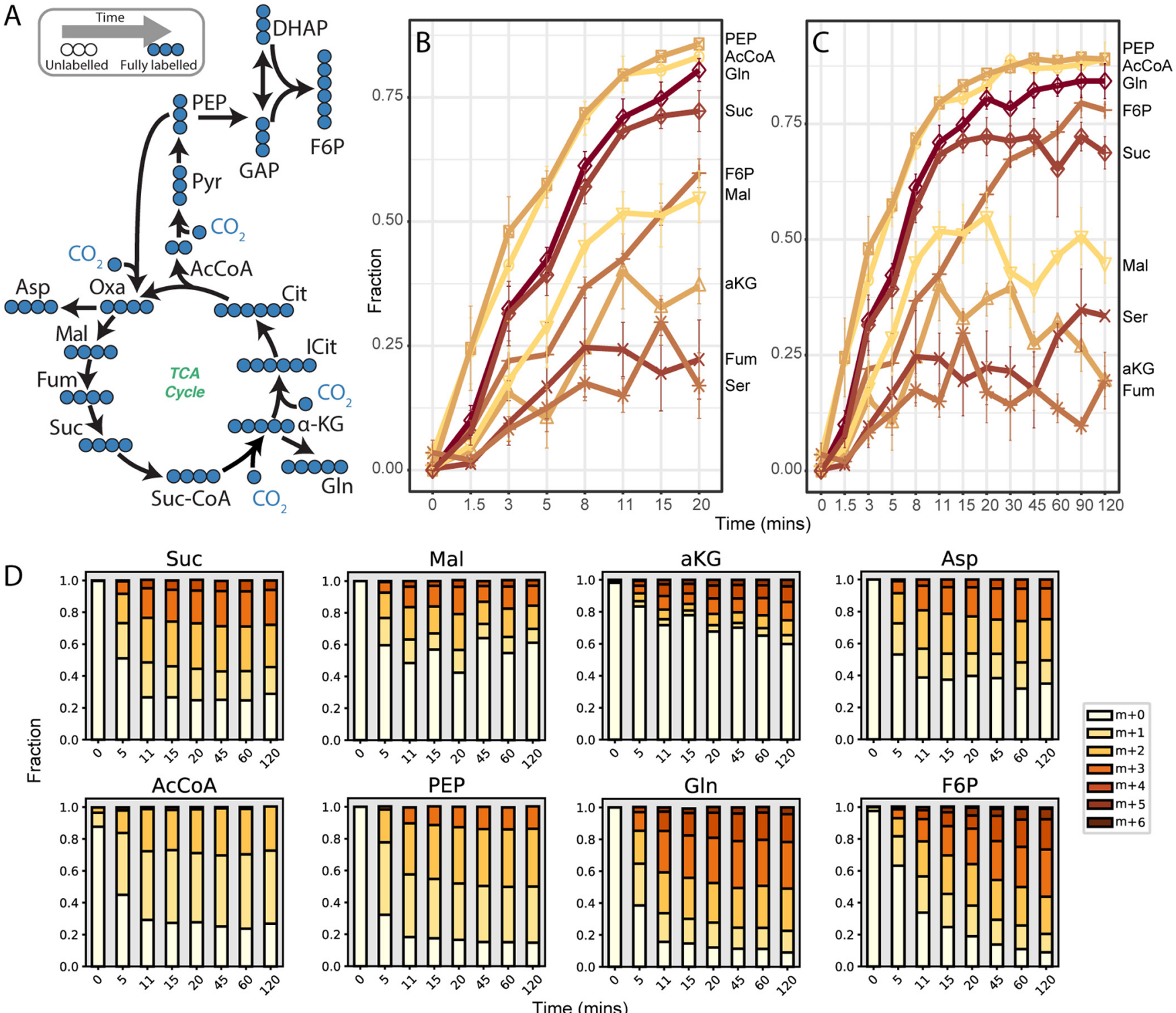

FIG 5 Dynamic ${ }^{13} \mathrm{C}$ labeling of selected central carbon metabolites during $\left[{ }^{13} \mathrm{C}\right]$ bicarbonate tracer experiments. (A) Expected ${ }^{13} \mathrm{C}$ labeling of central metabolism over time. ${ }^{13} \mathrm{C}$ enrichment of selected metabolites over 20 (B) and $120 \mathrm{~min}(\mathrm{C}) .{ }^{13} \mathrm{C}$ enrichment values were normalized to a tracer ${ }^{13} \mathrm{C}$ fraction of 1. (D) Mass isotopomer distributions for selected metabolites. AcCoA, acetyl-CoA, aKG, alpha-ketoglutarate, Asp, aspartic acid, F6P, fructose 6-phosphate, Fum, fumarate, Gln, glutamine, Mal, malate, PEP, phosphoenolpyruvate, Ser, serine, Suc, succinate. All measured metabolite mass isotopomer distributions can be found in Data Set S2.

In addition to the TCA cycle, fast labeling of 3-phosphoglycerate, fructose 6-phosphate, glucose 6-phosphate, and sedoheptulose 7-phosphate was observed (Fig. 5; Data Set S2). This is consistent with the use of gluconeogenesis and the pentose phosphate pathway for synthesis of precursor metabolites in N. moscoviensis.

Potential for formatotrophic and mixotrophic growth. It has been demonstrated that several Nitrospira species can use formate as an energy source and that their genomes contain a formate transporter (FocA) and a soluble $\mathrm{NAD}^{+}$-reducing formate dehydrogenase $(\mathrm{FDH})$, which catalyzes the oxidation of formate to $\mathrm{CO}_{2}$ with concomitant reduction of $\mathrm{NAD}^{+}$to $\mathrm{NADH}(5,9)$. Notably, N. moscoviensis and the marine Nitrospira marina were reported to grow less efficiently on formate than on nitrite $(5,9)$, a trend we confirmed for N. moscoviensis in batch experiments (see Fig. S4A). Furthermore, growth on formate was tested over a range of $\mathrm{pH}$ conditions, as previous studies indicated that formate oxidation may have a different $\mathrm{pH}$ optimum than nitrite oxidation 


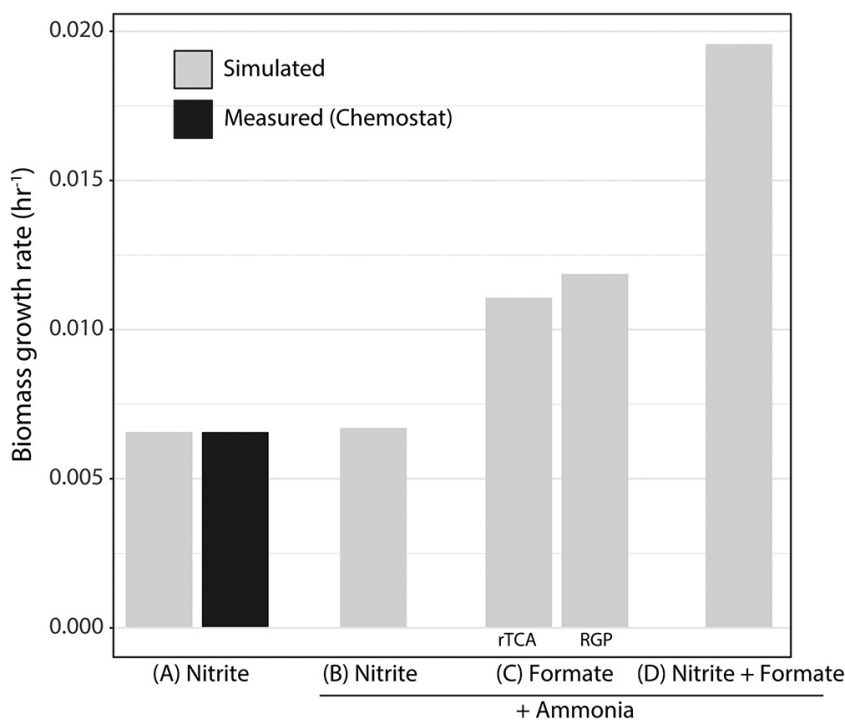

FIG 6 Model predictions for N. moscoviensis growth rate utilizing different substrates (nitrite, formate, and formate plus nitrite) and different formate assimilation pathways. rTCA, reductive TCA cycle; RGP, reductive glycine pathway. The substrate uptake fluxes in each scenario were as follows: nitrite, $8.5 \mathrm{mmol}$ $\mathrm{g}$ dry weight ${ }^{-1} \mathrm{~h}^{-1}$; formate, $0 \mathrm{mmol} \mathrm{g}$ dry weight ${ }^{-1} \mathrm{~h}^{-1}$ (A); nitrite, $8.7 \mathrm{mmol} \mathrm{g}$ dry weight ${ }^{-1} \mathrm{~h}^{-1}$; formate, $0 \mathrm{mmol} \mathrm{g}$ dry weight ${ }^{-1} \mathrm{~h}^{-1}(\mathrm{~B})$; nitrite, $0 \mathrm{mmol} \mathrm{g}$ dry weight ${ }^{-1} \mathrm{~h}^{-1}$; formate, $6.0 \mathrm{mmol} \mathrm{g}^{-1}$ dry weight ${ }^{-1} \mathrm{~h}^{-1}$ (C); nitrite, $8.7 \mathrm{mmol} \mathrm{g}$ dry weight ${ }^{-1} \mathrm{~h}^{-1}$; formate, $6.0 \mathrm{mmol} \mathrm{g}$ dry weight ${ }^{-1} \mathrm{~h}^{-1}$ (D). Scenario A substrate uptake fluxes were based on measured values from chemostat experiments conducted in this study; scenarios $B$ to $D$ substrate uptake fluxes were based on measured values from batch experiments performed by Koch et al. (5) (provided in Fig. S4). In scenario C, the RGP was inactivated by setting the flux through the formate-tetrahydrofolate ligase reaction (rxn00690_c0) to zero. Model reactions, compounds, and FBA solutions can be found in Data Set 1.

$(25,26)$. However, growth with formate as the sole energy source was even less efficient at lower $\mathrm{pH}$ values (Fig. S4B), indicating that the low growth efficiency on formate at $\mathrm{pH} 7.7$ was not due to a difference in $\mathrm{pH}$ optima for formate oxidation.

Additionally, we used the model to explore predicted growth efficiency and metabolism of $N$. moscoviensis on formate compared to those under chemolithoautotrophic conditions. Comparisons were performed on minimal NOB medium plus ammonia with the following substrates: (i) nitrite and $\mathrm{O}_{2}$, (ii) formate and $\mathrm{O}_{2}$, and (iii) nitrite plus formate and $\mathrm{O}_{2}$. The nitrite uptake rate was derived from chemostat measurements, whereas the formate uptake rate was estimated from data provided by Koch et al. (5) (Fig. S4A). In contrast to the reported slower growth on formate, the model predicted that formate or formate plus nitrite utilization would increase the growth rate of $N$. moscoviensis by approximately 1.9- or 3.0-fold, respectively (Fig. 6). Here, electrons derived from formate oxidation were predicted to drive energy conservation via the electron transport chain while also providing reducing equivalents for biosynthesis. A possible mechanism to explain the slower growth of $N$. moscoviensis on formate could be that complex I is constrained or limited in the oxidative direction, as this enzyme normally functions reductively to generate NADH via reverse electron flow in Nitrospira (3). Under such a scenario, a highly active FDH would generate reduction equivalents from formate oxidation (i.e., NADH) faster than their oxidation by the electron transport chain, resulting in an electron imbalance that would have to be alleviated by, for example, the secretion of a reduced product. We used the model to test this hypothesis by fixing the formate oxidation rate to the measured value and constraining flux through complex I to the measured growth rate (based on rates by Koch et al. [5], provided in Fig. S4A). Indeed, this resulted in model solutions that could balance electrons via several different central carbon reactions of the TCA cycle and/or amino acid biosynthesis followed by product secretion (Data Set S1, "FBA solutions"). However, this hypothesis remains untested and requires experimental investigation.

Besides its function as an energy source, formate has also been shown to be used as a carbon source by uncultured Nitrospira species (10), although the pathway for assimilation 

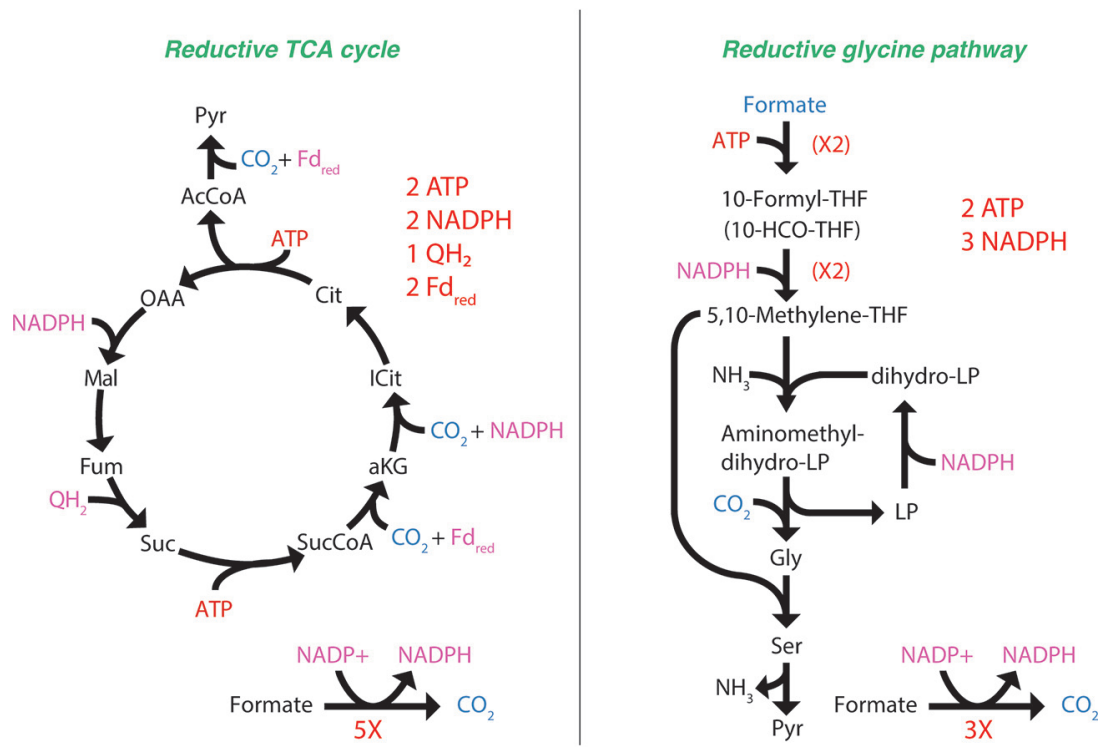

FIG 7 Comparison of the reductive TCA cycle and the reductive glycine pathway.

currently remains unclear. Metabolic reconstruction suggested that formate could potentially be assimilated either indirectly through oxidation to $\mathrm{CO}_{2}$ and subsequent assimilation via the rTCA cycle or directly via the reductive glycine pathway (27), for which all proteins are encoded in the genome (Data Set S1; Table S2). We thus examined the growth benefits of using either the rTCA cycle or the reductive glycine pathway for formate assimilation by turning on/off key reactions in the model. FBA predicted that $N$. moscoviensis growth rates would improve approximately $4 \%$ by directly assimilating formate via the reductive glycine pathway versus that indirectly via the rTCA cycle (Fig. 6). This is because the reductive glycine pathway consumes less energy than the rTCA cycle, as low-potential electron carriers (i.e., reduced ferredoxin) are not required, and in total, four electrons less are required per pyruvate molecule formed from formate instead of $\mathrm{CO}_{2}$ (Fig. 7). In this scenario, approximately $6 \%$ of the formate would be directly assimilated via the reductive glycine pathway rather than oxidizing all formate to $\mathrm{CO}_{2}$ followed by reassimilation (Data Set $\mathrm{S} 1$ "FBA solutions").

$\left[{ }^{13} \mathrm{C}\right]$ formate tracer experiments suggest formate is indirectly assimilated via $\mathbf{C O}_{\mathbf{2}}$ fixation. To experimentally determine the assimilation pathway of formate in $\mathrm{N}$. moscoviensis, isotopic tracer experiments with $\left[{ }^{13} \mathrm{C}\right]$ formate were performed with batch cultures in sealed serum bottles. Cultures were first acclimated to growth on $0.5 \mathrm{mM}$ unlabeled formate without the presence of nitrite for $24 \mathrm{~h}$. Subsequently, $1 \mathrm{mM}\left[{ }^{13} \mathrm{C}\right]$ formate was added to the cultures, and intracellular metabolome and gas headspace samples were collected before addition of $\left[{ }^{13} \mathrm{C}\right]$ formate and after 15, 30, 60, 180, and $300 \mathrm{~min}$ for isotopic analysis. Consistent with $\left[{ }^{13} \mathrm{C}\right]$ formate oxidation to $\mathrm{CO}_{2}$, continuous production of ${ }^{13} \mathrm{CO}_{2}$ in the headspace gas was observed during the experiment (Fig. 8A). Intracellular formate was measured to be $\sim 40 \%{ }^{13} \mathrm{C}$ enriched from 15 min onwards, consistent with formate transport into the cell (Fig. 8B). However, the majority of measured metabolites had no detectable ${ }^{13} \mathrm{C}$ enrichment, expect for succinate, glutamate, glutamine, and fructose 6phosphate (Data Set S2; Fig. 8B). Moreover, mass isotopomer distributions for these metabolites showed a consistent increase in labeled carbons overtime (Fig. 8B), similar to results from $\left[{ }^{13} \mathrm{C}\right]$ bicarbonate experiments (Fig. 3). Together, these results suggest that formate was being oxidized to $\mathrm{CO}_{2}$ and then assimilated via the rTCA cycle and that the reductive glycine pathway is not active in N. moscoviensis.

\section{DISCUSSION}

Previous studies have shown the utility of constraint-based reconstruction and analysis for exploring the metabolic capabilities of microorganisms that have important 

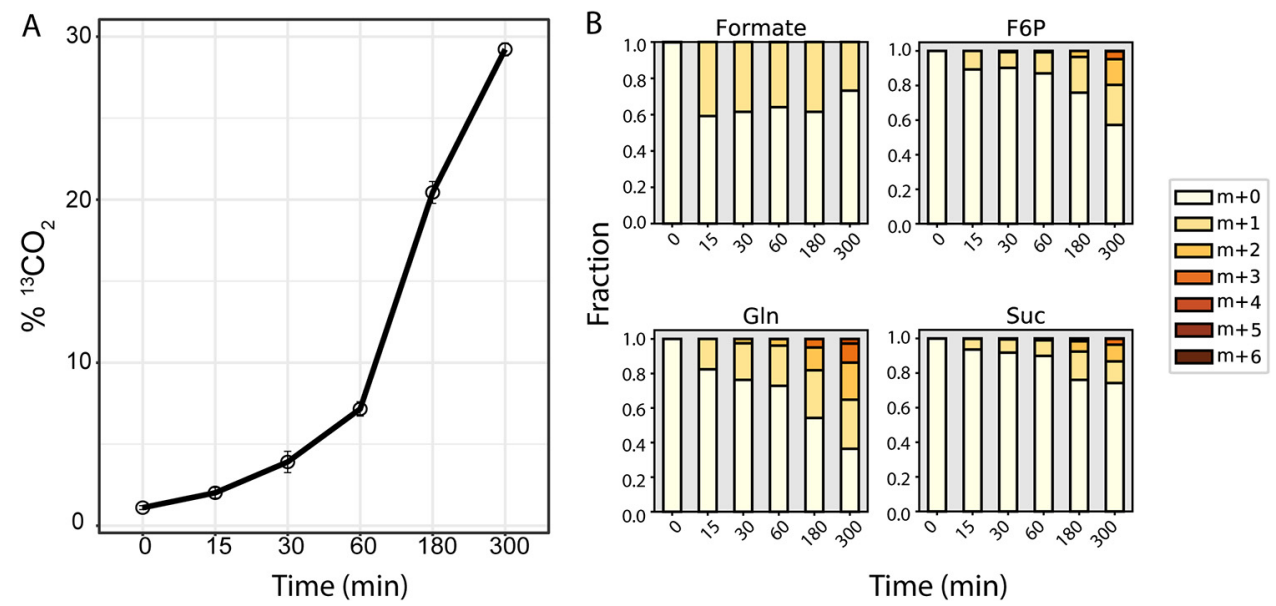

FIG 8 Oxidation and assimilation of $\left[{ }^{13} \mathrm{C}\right]$ formate by N. moscoviensis. (A) Production of ${ }^{13} \mathrm{CO}_{2}$ from $\left[{ }^{13} \mathrm{C}\right]$ formate oxidation. (B) Mass isotopomer distributions for selected metabolites during batch $\left[{ }^{13} \mathrm{C}\right]$ formate tracer experiments. All measured metabolite mass isotopomer distributions can be found in Data Set S2.

environmental roles (28-30). The model (iNmo686) provides a framework for examining Nitrospira metabolism at a systems level and will serve as a knowledge base that can be continually refined to drive understanding and improve predictions of Nitrospira ecophysiology. Our analysis shows that the model quantitatively predicts $N$. moscoviensis chemolithoautotrophic growth on nitrite. Flux balance analysis provided estimates for the amount of nitrite used for energy conservation and $\mathrm{CO}_{2}$ fixation into biomass, offering a quantitative model for linking catabolism and anabolism. The model also accurately provides quantitative estimates of carbon and electron flux distribution, reinforcing the metabolic network predicted based on the proteomic measurements from this study and from previous genomic and transcriptomic analyses of Nitrospira $(3,13)$.

${ }^{13} \mathrm{C}$ labeling metabolomic analysis revealed a complex picture for central carbon metabolism in Nitrospira. While our results validated that Nitrospira uses the rTCA cycle for $\mathrm{CO}_{2}$ fixation during chemolithoautotrophic growth, ${ }^{13} \mathrm{C}$ enrichment values revealed extensive unlabeled pools of central carbon intermediates in the metabolome (Fig. 5). Because all ${ }^{13} \mathrm{C}$ enrichment values increased monotonically, we reasoned that turnover of carbon storage reserves or macromolecules was not responsible for this observation. However, compartmentalization of metabolism or substrate channeling may offer a possible explanation. In cells that contain multiple pools of the same metabolite (e.g., cells with mitochondria), differences in their labeling will result in an aggregated measurement of the mixed pool because they are extracted together. If only one of those pools is actively participating in metabolism, the aggregation of both labeled and unlabeled pools will serve to dilute the overall ${ }^{13} \mathrm{C}$ enrichment. While electron micrograph images suggest that no intracytoplasmic membranes or carboxysomes are present in $N$. moscoviensis cells $(6,31,32)$, it is possible that separate metabolite pools may arise from certain enzymes/pathways having different subcellular locations within the cytoplasm (33). However, this requires further investigation.

An alternative and maybe more likely mechanism to explain the low ${ }^{13} \mathrm{C}$ enrichment levels of some metabolites is substrate channeling, the direct passing of pathway intermediates between enzyme active sites without escaping into the cytoplasm, facilitated by noncovalent dynamic enzyme complexes $(34,35)$. This can result in lower-thanexpected ${ }^{13} \mathrm{C}$ enrichment values due to "leaked" metabolites that create a separate cytoplasmic pool with a much slower turnover rate $(34,35)$. Given that several metabolites immediately downstream of the rTCA cycle (aspartate, glutamate, and glutamine) were more strongly labeled than their precursor TCA intermediates, we 
suspect that rTCA cycle enzymes may interact to form a supramolecular complex, or metabolon, to efficiently transport reactants between enzyme active sites. This brings the advantage that high local substrate concentrations enable higher pathway fluxes, and intermediates can be protected from the bulk phase, limiting competition between competing pathways and protecting the cell from toxicity $(34,36)$. Given the significant thermodynamic barriers encountered in the rTCA by the consecutive operation of alpha-ketoglutarate synthase and isocitrate dehydrogenase $(\Delta G>40 \mathrm{~kJ} / \mathrm{mol})$ as well as pyruvate synthase $(\Delta G>14 \mathrm{~kJ} / \mathrm{mol})(21)$, substrate channeling maybe be important for overcoming these unfavorable reactions in addition to their indirect coupling to ATP hydrolysis via succinyl-CoA synthetase and ATP citrate lyase. Further confirmation of this channeling could be obtained through in vivo cross-linking coupled to proteomic analysis, as has been done to investigate the malate dehydrogenase-citrate synthase-aconitase complex of the oxidative TCA cycle in other organisms $(23,24)$.

In addition to chemolithoautotrophic growth on nitrite, genome-scale modeling allowed us to assess different hypotheses regarding formatotrophic and mixotrophic growth of $N$. moscoviensis. Nitrospira species have been observed to oxidize formate to $\mathrm{CO}_{2}$ for energy conservation under laboratory conditions (5) and also to assimilate formate in situ during wastewater treatment (10). However, while our analysis predicted that $N$. moscoviensis should grow 2 to 3 times faster on formate than on nitrite, batch experiments confirmed the previously reported reduced growth efficiency of $\mathrm{N}$. moscoviensis on formate (5).

Interestingly, N. marina also showed poor growth with formate compared to that with nitrite (9), even though it possesses a cytoplasmic formate dehydrogenase that is only distantly related to the one of $N$. moscoviensis. Therefore, it is tempting to speculate that poor growth on formate might be a general feature of Nitrospira caused by its overall metabolism. Our modeling analysis suggested that one possible mechanism to explain this might be that the rate of formate oxidation exceeds the rate that electrons can flow through the electron transport chain, resulting in $\mathrm{NADH}$ accumulation that requires electrons to be segregated via secreted carbon metabolites, diverting carbon from cell growth. A possible alternative explanation for inefficient growth on formate might be an increased production of reactive oxygen species (ROS) by a respiratory chain that is potentially not well adapted for electron flow in the oxidative direction. The metabolic cost for increased ROS defense might result in low growth efficiency. However, the exact metabolic mechanism resulting in poor growth on formate awaits further physiological analysis. In addition, it also remains to be tested whether all Nitrospira species grow slower using formate as an electron donor and whether other environmental conditions can boost formatotrophic growth.

Genome-scale modeling further allowed us to generate hypotheses on the use of formate as a carbon source for $N$. moscoviensis anabolism. While modeling predicted that small growth improvements would be achieved by directly assimilating formate via the reductive glycine pathway, in vivo $\left[{ }^{13} \mathrm{C}\right]$ formate tracer experiments demonstrated that $N$. moscoviensis adheres to its autotrophic lifestyle, assimilating $\mathrm{CO}_{2}$ derived from formate oxidation via the rTCA cycle. This may be an evolutionary adaptation to avoid energetic costs associated with remodeling its proteome in environments where formate may only become transiently available, for example, at oxic-anoxic interfaces common to the habitats of Nitrospira (1). Together with the poor growth on formate, this observation highlights the importance of validating model predictions with experimental measurements.

In conclusion, our work provides the first genome-scale reconstruction and analysis of Nitrospira metabolism, offering unique insights on its versatile ecophysiology. The genome-scale model (iNmo686) provides quantitative estimates of chemolithoautotrophic growth and pathway fluxes and serves as a valuable tool for hypothesis-driven discovery. Our ${ }^{13} \mathrm{C}$ labeling metabolomic results also provide the first insights on Nitrospira's in vivo central carbon metabolism, confirming the high activity of the rTCA 
cycle for $\mathrm{CO}_{2}$ fixation. Future efforts to combine ${ }^{13} \mathrm{C}$ metabolomics with genome-scale modeling should provide a valuable approach for quantitatively understanding the regulation of Nitrospira metabolism under different environmental conditions and microbe-microbe interactions. This will further expand the systems biology framework developed in this study, ultimately leading to the systematic prediction and control of Nitrospira metabolism in natural and engineered ecosystems.

\section{MATERIALS AND METHODS}

Cultivation of $\boldsymbol{N}$. moscoviensis cells. $N$. moscoviensis $\mathrm{M}-1$ was grown in NOB mineral salts medium for lithoautotrophic growth as described in Spieck and Lipski (37) except that $\mathrm{CaCO}_{3}$ was replaced with $\mathrm{CaCl}_{2} \cdot 2 \mathrm{H}_{2} \mathrm{O}$ at the same concentration, and the following trace element composition was used per liter of medium: $34.4 \mu \mathrm{g}$ of $\mathrm{MnSO}_{4} \cdot 1 \mathrm{H}_{2} \mathrm{O}, 50 \mu \mathrm{g}$ of $\mathrm{H}_{3} \mathrm{BO}_{3}, 70 \mu \mathrm{g}$ of $\mathrm{ZnCl}_{2}, 72.6 \mu \mathrm{g}$ of $\mathrm{Na}_{2} \mathrm{MoO}_{4} \cdot 2 \mathrm{H}_{2} \mathrm{O}, 20 \mu \mathrm{g}$ of $\mathrm{CuCl}_{2} \cdot 2 \mathrm{H}_{2} \mathrm{O}, 24 \mu \mathrm{g}$ of $\mathrm{NiCl}_{2} \cdot 6 \mathrm{H}_{2} \mathrm{O}, 80 \mu \mathrm{g}$ of $\mathrm{CoCl}_{2} \cdot 6 \mathrm{H}_{2} \mathrm{O}$, and $2,000 \mu \mathrm{g}$ of $\mathrm{FeSO}_{4} \cdot 7 \mathrm{H}_{2} \mathrm{O}$. Nitrilotriacetic acid was added equimolar to all trace elements as a complexing agent.

N. moscoviensis was cultivated in a 7-liter membrane bioreactor (MBR) inoculated with an active batch culture and operated as described by Mundinger et al. (13). The working volume of the reactor was 3 liters and included $\mathrm{pH}$, dissolved oxygen, temperature, and level controls (all by Applikon Biotechnology B.V., Schiedam, The Netherlands). The bioreactor was continuously sparged with $\mathrm{Ar} / \mathrm{CO}_{2}$ (95\%:5\% [vol/vol]) and air at a rate of $10 \mathrm{ml} / \mathrm{min}$ to maintain a dissolved oxygen concentration of $\sim 30 \%$. $\mathrm{pH}$ was controlled at 7.7 using a $1 \mathrm{M} \mathrm{KHCO}_{3}$ buffer. Temperature was maintained at $39^{\circ} \mathrm{C}$ using a looptype heat exchanger, and the reactor was continuously stirred at $150 \mathrm{rpm}$. Reactor and all cultivation media and solutions were sterilized by autoclaving or sterile filtration prior to use, and the reactor was operated aseptically to maintain culture purity. Nitrite and nitrate concentrations were check daily to ensure all nitrite was consumed stoichiometrically to nitrate (Nitrite test strips MQuant; Merck, Darmstadt, Germany) and that nitrite was always limiting. Cultures were maintained in steady-state growth at a dilution rate of $0.006 \mathrm{~h}^{-1}$ and a substrate feeding rate of 2 to $2.5 \mathrm{mmol} \mathrm{NO}_{2}{ }^{-}$liter $^{-1}$ day $^{-1}$.

Genome-scale model reconstruction and analysis. The genome-scale metabolic model of N. moscoviensis (iNmo686) was reconstructed from the NCBI's genome sequence for $N$. moscoviensis (accession number NZ_CP011801.1) using the ModeISEED pipeline (38) implemented in KBase (16), followed by manual curation using the MetaCyc database (15) and available literature $(1,3,5)$. The model was gap filled manually through the addition of reactions not annotated in the genome to ensure that all biomass components could be produced on NOB minimal medium. Growth- and nongrowth-associated maintenance energy requirements were determined by plotting experimentally measured nitrite uptake rates as a function of the growth rate set during steady-state bioreactor cultivation (39). The biomass equation was derived from biomass composition measurements of $N$. moscoviensis (see Data Set S1 in the supplemental material). The model was formulated in Systems Biology Markup Language (SBML) level 3 version 1.0 and is available at GitHub (https://github.com/celawson87/Nitrospira-moscoviensis -GEM). Flux balance analysis was used to simulate in silico growth by solving the linear program:

$$
\begin{gathered}
\text { Max } v_{\text {biomass, }} \\
\text { s.t., } \\
S \times v=0, \\
v_{\min } \leq v \leq v_{\max },
\end{gathered}
$$

where $v_{\text {biomass }}$ is the flux through the biomass objective function, $S$ is the stoichiometric matrix generated from the reconstruction with rows representing metabolites, columns representing reactions, and entries representing metabolite stoichiometric coefficients, $v$ is the vector of steady-state reaction fluxes, and $v_{\min }$ and $v_{\max }$ are the minimum and maximum allowable reaction fluxes, respectively. Flux balance analysis was performed in Python version 3.7.2 using the COBRApy package (40).

Biomass composition analysis. Cultures were centrifuged $\left(10,000 \mathrm{rpm}, 15 \mathrm{~min}, 4^{\circ} \mathrm{C}\right)$ to obtain cell pellets, which were subsequently freeze-dried prior to analysis. Total protein concentration was determined using the Pierce bicinchoninic acid (BCA) protein assay kit (Thermo Fisher Scientific), and amino acid composition was determined according to Carnicer et al. (41) using a Varian 920-LC high-performance liquid chromatography amino acid analyzer. Total carbohydrates were determined using the phenol-sulfuric acid method (42). Total lipid content was determined via the sulfo-phospho-vanillin reaction (43), and the lipid composition for N. moscoviensis was that reported by Lipski et al. (20). Total RNA and DNA content was determined according to Benthin et al. (44). Total inorganic content was determined by combustion of freeze-dried biomass in an oven at $550^{\circ} \mathrm{C}$ for $12 \mathrm{~h}$. Lipid headgroup composition, ion composition, and soluble pool composition were derived from the Escherichia coli biomass equation reported for IAF 1260 (45).

${ }^{13} \mathrm{C}$ isotopic tracer experiments. ${ }^{13} \mathrm{C}$-labeled sodium bicarbonate was rapidly introduced (within 1 $\mathrm{min}$ ) into the bioreactor containing N. moscoviensis cells growing under steady-state conditions to a final concentration of approximately $30 \mathrm{mM}$. Following ${ }^{13} \mathrm{C}$-label introduction, samples were rapidly withdrawn from the reactor at time points $0,1.5,3,5,8,11,15,20,30,45,60,90$, and $120 \mathrm{~min}$. Samples were immediately filtered (Millipore $0.45-\mu \mathrm{m}$ hydrophilic nylon filter HNWPO4700) using a vacuum pump to remove extracellular medium, and filters were placed face down in $1.5 \mathrm{ml}$ of $-80^{\circ} \mathrm{C}$ extraction solvent (40:40:20 acetonitrile-methanol-water) for cell quenching and metabolite extraction. Samples were then 
centrifuged $\left(10,000 \mathrm{rpm}, 4^{\circ} \mathrm{C}, 5 \mathrm{~min}\right)$, and $1 \mathrm{ml}$ of cell-free supernatant was collected and stored at $-80^{\circ} \mathrm{C}$ for metabolomic analysis. The time zero sample corresponded to the period directly before ${ }^{13} \mathrm{C}$ label addition. The ratio of ${ }^{13} \mathrm{C}$ to ${ }^{12} \mathrm{C}$ dissolved inorganic carbon remained constant during the course of the 2-h experimental period as determined by gas chromatography-mass spectrometry (GC-MS) analysis as described below.

Formate batch experiments. $N$. moscoviensis cells were harvested from the membrane bioreactor. The biomass was centrifuged at $8,000 \times g$ for $15 \mathrm{~min}$ at $25^{\circ} \mathrm{C}$ and washed by resuspending the cells in fresh mineral NOB medium. This was repeated until no nitrite or nitrate was detectable via nitrite/nitrate test strips (Nitrite test strips MQuant; Merck) in the culture. Subsequently, the cells were transferred to sterile $120-\mathrm{ml}$ serum bottles (triplicate bottles per time point, 6 time points total) containing $50 \mathrm{ml} \mathrm{NOB}$ mineral salts medium with $0.5 \mathrm{mM}$ sodium formate and no nitrite but $0.187 \mathrm{mM} \mathrm{NH}_{4} \mathrm{Cl}$ as the nitrogen source. Bottles were crimp sealed with a rubber stopper to allow monitoring of the gas headspace and were incubated at $39^{\circ} \mathrm{C}$ in the dark. Following $24 \mathrm{~h}$ of acclimation, $1 \mathrm{mM}{ }^{13} \mathrm{C}$-labeled sodium formate (Cambridge Isotopes Laboratories, MA, USA) was added to all incubations. At each time point (before addition and after $15,30,60,180$, and $300 \mathrm{~min}$ ), the isotopic composition of the gas headspace was measured using GCMS (see below), and subsequently, all 3 bottles corresponding to a given time point were sacrificed for metabolomics analysis. Bottle contents were filtered immediately using a vacuum pump, and metabolites were extracted using $-80^{\circ} \mathrm{C}$ extraction solvent (40:40:20 acetonitrile-methanol-water) as described above.

For growth experiments, $\mathrm{N}$. moscoviensis cells from the membrane bioreactor were harvested and washed until no nitrite or nitrate was detectable via nitrite/nitrate test strips (Nitrite test strips MQuant; Merck) (see above). The cells were transferred to sterile $120-\mathrm{ml}$ serum bottles with $50 \mathrm{ml}$ NOB mineral medium containing $0.187 \mathrm{mM} \mathrm{NH}_{4} \mathrm{Cl}$ as the nitrogen source and a pH adjusted to $6.6,7$, or 7.7 with $1 \mathrm{M} \mathrm{KHCO}_{3}$ and were sealed with rubber stoppers. The experiment was performed in duplicates (per pH value and substrate), and $5 \mathrm{mM}$ sodium nitrite or $5 \mathrm{mM}$ sodium formate was added to the incubations as the sole energy source. Growth was monitored for 7 days using optical density measurements at a wavelength of $600 \mathrm{~nm}$.

Metabolomic analysis. Samples were analyzed using a high-performance liquid chromatography (HPLC)-MS system consisting of a Vanquish UHPLC system (Thermo Scientific) coupled with electrospray ionization (ESI; negative polarity) to a hybrid quadrupole high-resolution mass spectrometer (Q Exactive Orbitrap; Thermo Scientific) operated in full scan mode for the detection of targeted compounds based on their accurate masses. Properties of full MS-selected ion monitoring (SIM) included a resolution of 140,000 , automatic gain control (AGC) target of 1E6, maximum isolation time (IT) of $40 \mathrm{~ms}$, and scan range from 70 to $1,000 \mathrm{~m} / \mathrm{z}$. LC separation was achieved using an ACQUITY UPLC BEH $C_{18}$ column (2.1by $100-\mathrm{mm}$ column, 1.7- $\mu \mathrm{m}$ particle size, part no. 186002352, serial number 02623521115711; Waters). Solvent A was $97 / 3$ water-methanol with $10 \mathrm{mM}$ tributylamine (TBA) adjusted to $\mathrm{pH} 8.1$ to 8.2 with $9 \mathrm{mM}$ acetic acid. Solvent $B$ was $100 \%$ methanol. Total run time was $25 \mathrm{~min}$ with the following gradient: $0 \mathrm{~min}$, $5 \%$ B; $2.5 \mathrm{~min}, 5 \%$ B; $5 \mathrm{~min}, 20 \%$ B; $7.5 \mathrm{~min}, 20 \%$ B; $13 \mathrm{~min}, 55 \%$ B; $15.5 \mathrm{~min}, 95 \%$ B; $18.5 \mathrm{~min}, 95 \%$ B; $19 \mathrm{~min}, 5 \% \mathrm{~B}$; and $25 \mathrm{~min}, 5 \% \mathrm{~B}$. Flow rate was $200 \mu \mathrm{Imin}^{-1}$. The autosampler and column temperatures were $4^{\circ} \mathrm{C}$ and $25^{\circ} \mathrm{C}$, respectively. Mass isotopomer distributions were corrected for natural abundance using the method of Su et al. (46), and ${ }^{13} \mathrm{C}$ enrichment values were calculated using the formula $(1 / N) \sum_{i=1}^{N} M i \times i$, where $N$ is the number of carbon atoms in the metabolite and $M i$ is the fractional abundance of the ith mass isotopomer.

GC-MS analysis of dissolved inorganic carbon isotopic fractions. Isotopic fractions of dissolved inorganic carbon in the liquid media were measured based on a modified headspace method (47). Three milliliters of liquid culture was collected from the bioreactor with a syringe and directly filtered through a $0.45-\mu \mathrm{m}$ filter and 26-gauge needle into a $120-\mathrm{ml}$ bottle containing $1 \mathrm{ml} 6 \mathrm{M} \mathrm{HCl}$ (strong acid) crimp sealed with a rubber stopper. Prior to adding the liquid sample, bottles and $\mathrm{HCl}$ were flushed with either $100 \% \mathrm{~N}_{2}$ or $\mathrm{Ar}$ gas to void the headspace of background $\mathrm{CO}_{2}$. Samples were equilibrated with the acid in the bottles for at least $1 \mathrm{~h}$ at room temperature to drive all dissolved inorganic carbon into the gas phase. Fifty-microliter gas samples were then collected in a gas-tight syringe with a needle (Hamilton) from the bottle's headspace and the isotopic fractions of ${ }^{12} \mathrm{CO}_{2}$ and ${ }^{13} \mathrm{CO}_{2}$ were determined using a gas chromatograph (Agilent 6890 equipped with $6 \mathrm{ft}$ Porapak $\mathrm{Q}$ and molecular sieve columns) coupled to a mass spectrometer (GC-MS) (Agilent 5975C GC MSD; Agilent, Santa Clara, CA).

Large-scale discovery proteomics of whole-cell and membrane fractions. (i) Whole-cell fraction sample preparation and proteolytic digestion. Biomass was harvested by centrifugation, washed once with Tris-EDTA buffer, $\mathrm{pH} 7.7$, and frozen using liquid $\mathrm{N}_{2}$. Cell pellets were stored at $-80^{\circ} \mathrm{C}$ until further processing. For protein extraction, cell pellets $(445 \mathrm{mg}$ wet weight) were resuspended in $5 \mathrm{ml}$ $50 \mathrm{mM}$ triethylammonium bicarbonate (TEAB) buffer, $1 \%$ sodium deoxycholate, $\mathrm{pH}$ 8, and mixed 1:1 with B-PER reagent (Thermo Fisher Scientific, Waltham, MA, USA). Cells were lysed by two rounds of sonification (Sonifier B-12 with microtip; Branson Sonic Power Company, Danbury, CT, USA) at setting 6 for $30 \mathrm{~s}$, intermitted by cooling on ice. Cell debris and unopened cells were separated from the sample by centrifugation $(14,000 \times g$, room temperature [RT], $10 \mathrm{~min})$. Proteins were precipitated by addition of $250 \mu \mathrm{l} 100 \%$ (wt/vol) trichloroacetic acid (TCA) to $1 \mathrm{ml}$ of sample and incubation for $20 \mathrm{~min}$ on ice. Proteins were collected by centrifugation $(14,000 \mathrm{rpm}, \mathrm{RT}, 5 \mathrm{~min})$ and washed twice with ice-cold acetone. Next, the protein pellet was resuspended in $400 \mu \mathrm{l} 50 \mathrm{mM}$ TEAB buffer aided by a heating step at $40^{\circ} \mathrm{C}$ for $1 \mathrm{~h}$ with intermittent vortexing. One hundred microliters of the protein extract was diluted 5 times with $6 \mathrm{M}$ urea, $200 \mathrm{mM}$ ammonium bicarbonate (ABC) buffer followed by vortexing and a heating step for $20 \mathrm{~min}$ at $45^{\circ} \mathrm{C}$ at $1,450 \mathrm{rpm}$ and $25 \mathrm{~min}$ of sonification. Acetonitrile (ACN) was added to a final concentration of $50 \%$, followed by another heating step. A $200-\mu$ l aliquot of the sample was reduced by 
the addition of dithiothreitol (DTT) to a final concentration of $2.3 \mathrm{mM}$ followed by incubation at $37^{\circ} \mathrm{C}$ for $1 \mathrm{~h}$. Next, the sample was alkylated by the addition of iodoacetamide (IAM) to a final concentration of $3.75 \mathrm{mM}$ followed by incubation in the dark at room temperature for $30 \mathrm{~min}$. The sample was diluted with $200 \mathrm{mM} \mathrm{ABC}$ buffer to a concentration of urea of $<1 \mathrm{M}$. Lastly, a 150- $\mu$ l aliquot of the protein sample was digested with $5 \mu \mathrm{g}$ of sequencing-grade trypsin (Promega, Madison, WI, USA) overnight at $37^{\circ} \mathrm{C}$.

(ii) Membrane fraction sample preparation and proteolytic digestion. Biomass was harvested by centrifugation, washed once with Tris-EDTA (TE) buffer, and frozen using liquid $\mathrm{N}_{2}$. Cell pellets were stored at $-80^{\circ} \mathrm{C}$ until further processing. For protein extraction, cell pellets (1.95 g wet weight) were resuspended in $13 \mathrm{ml} 50 \mathrm{mM}$ TEAB buffer, $1 \%$ sodium deoxycholate, $\mathrm{pH} 8$, and mixed 1:1 with B-PER reagent (Thermo Fisher Scientific). Cells were lysed by three passages through a French press at $138 \mathrm{MPa}$ pressure. Cell debris and unopened cells were pelleted from the sample by centrifugation $(5,000 \times g$, $\left.4^{\circ} \mathrm{C}, 15 \mathrm{~min}\right)$. The membrane fraction was prepared using ultracentrifugation $\left(45,000 \mathrm{rpm}, 4^{\circ} \mathrm{C}, 1 \mathrm{~h}\right.$ on an Optima-90 ultracentrifuge; Beckman-Coulter, Brea, CA, USA) and washed once in 200 mM ABC buffer, followed by another ultracentrifugation step to collect the membrane pellet. Finally, the membrane pellet was resuspended in $400 \mu \mathrm{l} 200 \mathrm{mM} \mathrm{ABC}, 1 \% \mathrm{~N}$-dodecyl $\beta$-D-maltoside (DDM), and membrane proteins were solubilized overnight in an orbital shaker at $4^{\circ} \mathrm{C}$. The sample was clarified by centrifugation $(20,000 \times g, R T, 15 \mathrm{~min})$. Urea was added to the sample to a final concentration of $6 \mathrm{M}$. The sample was reduced by the addition of DTT to a final concentration of $2.3 \mathrm{mM}$ followed by incubation at $37^{\circ} \mathrm{C}$ for $1 \mathrm{~h}$. Next, the sample was alkylated by the addition of IAM to a final concentration of $3.75 \mathrm{mM}$, followed by incubation in the dark at RT for $30 \mathrm{~min}$. The sample was diluted to a concentration of $1 \mathrm{mg} / \mathrm{ml}$ with $200 \mathrm{mM} \mathrm{ABC}, 1 \% \mathrm{DDM}$, and $6 \mathrm{M}$ urea. Five aliquots of $100 \mu \mathrm{g}$ protein each were digested or doubly digested using $2 \mu \mathrm{g}$ of each selected proteinase; all digestion steps were performed at $37^{\circ} \mathrm{C}$. The following different proteinases and combinations were tested: (i) LysC (Pierce), 4 h; (ii) trypsin (Promega), 4 h; (iii) chymotrypsin (Pierce), overnight; (iv) LysC, $4 \mathrm{~h}$ followed by trypsin, overnight; and (v) trypsin, $4 \mathrm{~h}$ followed by chymotrypsin, overnight. In digests with trypsin and chymotrypsin, ACN was added to a final concentration of $20 \%$, and the concentration of urea in the sample was diluted to $\leq 1 \mathrm{M}$ by the addition of $A B C$ buffer.

(a) Solid phase extraction for membrane protein digest. Proteolytic digests were desalted using an Oasis HLB 96-well plate (Waters, Milford, MA, USA) according to the manufacturer's protocol. The purified peptide eluate was further dried using a speed-vacuum concentrator.

(b) Large-scale shotgun proteomics. All dried peptide fractions were resuspended in $\mathrm{H}_{2} \mathrm{O}$ containing $3 \%$ acetonitrile and $0.1 \%$ formic acid using mild vortexing. An aliquot of every sample corresponding to approximately 100 to $200 \mathrm{ng}$ protein digest was analyzed in duplicates using a one-dimensional shotgun proteomics approach (48). Briefly, $1 \mu \mathrm{l}$ of sample was injected to a nano-liquid chromatography system consisting of an EASY nano LC 1200, equipped with an Acclaim PepMap RSLC RP $C_{18}$ separation column $(50 \mu \mathrm{m}$ by $150 \mathrm{~mm}, 2 \mu \mathrm{m}$, and $100 \AA$ ), and a QE plus Orbitrap mass spectrometer (Thermo). The flow rate was maintained at $300 \mathrm{nl} / \mathrm{min}$ over a linear gradient using $\mathrm{H}_{2} \mathrm{O}$ containing $0.1 \%$ formic acid as solvent $\mathrm{A}$, and $80 \%$ acetonitrile in $\mathrm{H}_{2} \mathrm{O}$ and $0.1 \%$ formic acid as solvent $\mathrm{B}$. The soluble protein extract fractions were analyzed using a gradient from $5 \%$ to $30 \%$ solvent B over $90 \mathrm{~min}$, and finally to $75 \%$ B over $25 \mathrm{~min}$. The soluble membrane protein fractions were analyzed using a shorter gradient from $4 \%$ to $30 \%$ B over $32.5 \mathrm{~min}$ followed by a second step to $65 \%$ B over $12.5 \mathrm{~min}$, and data were acquired in total over $50 \mathrm{~min}$. In either case, the Orbitrap was operated in data-dependent acquisition mode acquiring peptide signals from 350 to 1,400 m/z at 70,000 resolution, where the top 10 signals were isolated from a window of $2.0 \mathrm{~m} / \mathrm{z}$ and fragmented using a normalized collision energy (NCE) of 30. The AGC target was set to $1 \mathrm{E} 5$, at a maximum IT of $54 \mathrm{~ms}$ and 17,500 resolution.

(c) Database search and data processing. Raw shotgun proteomics data from membrane proteins and soluble protein extract fractions were analyzed and combined using PEAKS Studio 8.5 (Bioinformatics Solutions Inc., Waterloo, Canada). Database search was performed allowing 20-ppm parent ion and 0.02Da fragment mass error tolerance. Search conditions further considered 3 missed cleavages for the respective enzymes used, carbamidomethylation as fixed and methionine oxidation and N/Q deamidation as variable modifications. Peptide spectra were matched against a $N$. moscoviensis sequence database (UniProt TrEMBL, June 2018, taxon identifier [Tax ID] 42253), which was modified by filtering out duplicated entries. The database search included the GPM cRAP contaminant database (https://www.thegpm .org/crap/) and a decoy fusion for determining false-discovery rates. Peptide spectrum matches were filtered against a $1 \%$ false-discovery rate (FDR), and protein identifications with 2 or more unique peptides were considered significant hits. For the prefractionated whole-cell proteome, proteins were counted if they were significantly identified in at least one of the subfractions.

Transmembrane helix predictions. The combined transmembrane topology and signal peptide prediction tool Phobius (http://phobius.sbc.su.se [49]) was employed for the prediction of membrane proteins. Since this tool is optimized to distinguish between transmembrane helices and signal peptides, we defined all proteins with a prediction of one or more transmembrane helices (TMHs) as membrane proteins.

Transcriptome analysis. To relate the proteome data to the previously published transcriptome of $N$. moscoviensis under stable cultivation conditions grown on nitrite (13), we reanalyzed the transcriptome data (GEO series accession number GSE123406) using the N. moscoviensis genome annotation version CP011801.1 as a reference, from which the UniProt protein sequences were derived (5). The transcriptomic reanalysis was performed as described by Mundinger et al. (13). In short, quality-filtered raw reads from lonTorrent PGM sequencing (minimum quality score of 0.05 , maximum sequencing length of $300 \mathrm{bp}$, allowing two ambiguous nucleotides) were mapped to the N. moscoviensis genome (NCBI accession number CP011801.1) using the mapping tool BBMap v35.92 (https://sourceforge.net/projects/ bbmap/) and counted using featureCounts (50) with the parameters minid-0.95 with fracOverlap-0.9 for 
a minimum alignment identity of $95 \%$ over $90 \%$ of the read length and ambig=random to assign reads with multiple top-scoring mapping locations randomly to a single location.

Since the genome includes several duplicated genes, the 4,790 protein coding sequences (CDSs) correspond to only 4,733 UniProt entries for nonidentical proteins. To directly compare transcription to proteome data, the transcriptome reads of those identical genes were thus summed up.

Expression levels of CDSs were compared by ranking them from high to low based on their reads per kilobase per million reads (RPKM) values. Additionally, the $\log _{2}$ fold to median was calculated for genome-wide visualizations of the gene expression levels in a Circos plot (51).

Data availability. The transcriptomic data set reanalyzed here can be found under the NCBI Gene Expression Omnibus data set accession number GSE123406.

The mass spectrometry proteomics raw data have been deposited to the ProteomeXchange Consortium (http://proteomecentral.proteomexchange.org) via the PRIDE partner repository (52) with the data set identifier PXD019583.

\section{SUPPLEMENTAL MATERIAL}

Supplemental material is available online only.

DATA SET S1, XLSX file, $0.2 \mathrm{MB}$.

DATA SET S2, XLSX file, 0.1 MB.

TEXT S1, PDF file, $0.1 \mathrm{MB}$.

FIG S1, JPG file, 0.1 MB.

FIG S2, PDF file, 0.4 MB.

FIG S3, PDF file, 0.9 MB.

FIG S4, PDF file, $0.3 \mathrm{MB}$.

TABLE S1, XLSX file, $0.9 \mathrm{MB}$.

TABLE S2, XLSX file, 0.1 MB.

TABLE S3, XLSX file, $0.1 \mathrm{MB}$.

\section{ACKNOWLEDGMENTS}

We thank Laura Hesp and Maartje van Kessel for assistance with the ${ }^{13} \mathrm{C}$-tracer experiments and Joshua Hamilton for helpful feedback on genome-scale model reconstruction.

Funding was provided by the National Science Foundation (CBET-1435661, CBET1803055, and MCB-1518130), the Netherlands Organization for Scientific Research (grants 016.Vidi.189.050, VI.Veni.192.086 and SIAM Gravitation grant 024.002.002), the European Research Council (ERC Advanced Grant Ecomom, 339880), a Wisconsin Distinguished Graduate Fellowship, a Postgraduate Scholarship-Doctoral (PGS-D) by the National Sciences and Engineering Research Council of Canada (NSERC), and the UW-Madison Office of the Vice Chancellor for Research and Graduate Education through the Microbiome Initiative. This research was performed in part using the Wisconsin Energy Institute computing cluster, which is supported by the Great Lakes Bioenergy Research Center as part of the U.S. Department of Energy Office of Science (DE-SC0018409). This material is based upon work supported by the U.S. Department of Energy, Office of Science, Office of Biological and Environmental Research, and Early Career Research Program under award number DE-SC0018998.

C.E.L., S.L., K.D.M., and D.R.N. designed the study. C.E.L. built the models, and C.E.L. and T.B.J. performed the metabolomic analysis. C.E.L., A.B.M., and H.K. performed the ${ }^{13} \mathrm{C}$ isotopic tracer experiments. A.B.M., M.P., and H.K. performed the proteomic analysis. H.K. performed the formate batch experiments. C.E.L. and C.A.W. performed the biomass compositional analysis. C.E.L. and A.B.M. wrote the manuscript. All authors provided valuable feedback and edits on the manuscript.

We declare no conflicts of interest.

\section{REFERENCES}

1. Daims H, Lücker S, Wagner M. 2016. A new perspective on microbes formerly known as nitrite-oxidizing bacteria. Trends Microbiol 24:699-712. https://doi.org/10.1016/j.tim.2016.05.004.

2. Daims H, Nielsen JL, Nielsen PH, Schleifer K-H, Wagner M. 2001. In situ characterization of nitrospira-like nitrite-oxidizing bacteria active in wastewater treatment plants. Appl Environ Microbiol 67:5273-5284. https://doi.org/10 .1128/AEM.67.11.5273-5284.2001.

3. Lücker S, Wagner M, Maixner F, Pelletier E, Koch H, Vacherie B, Rattei T, Damsté JSS, Spieck E, Le Paslier D, Daims H. 2010. A Nitrospira metagenome illuminates the physiology and evolution of globally important 
nitrite-oxidizing bacteria. Proc Natl Acad Sci U S A 107:13479-13484. https://doi.org/10.1073/pnas.1003860107.

4. Koch $H$, Galushko A, Albertsen M, Schintlmeister A, Spieck E, Richter A, Nielsen PH, Wagner M, Daims H, Gruber-Dorninger C, Lücker S, Pelletier E, Le Paslier D, Spieck E, Richter A, Nielsen PH, Wagner M, Daims H. 2014. Growth of nitrite-oxidizing bacteria by aerobic hydrogen oxidation. Science 345:1052-1054. https://doi.org/10.1126/science.1256985.

5. Koch H, Lücker S, Albertsen M, Kitzinger K, Herbold C, Spieck E, Nielsen $\mathrm{PH}$, Wagner M, Daims H. 2015. Expanded metabolic versatility of ubiquitous nitrite-oxidizing bacteria from the genus Nitrospira. Proc Natl Acad Sci U S A 112:11371-11376. https://doi.org/10.1073/pnas.1506533112.

6. Ehrich S, Behrens D, Lebedeva EV, Ludwig W, Bock E. 1995. A new obligately chemolithoautotrophic, nitrite-oxidizing bacterium, nitrospiramoscoviensis sp-nov and its phylogenetic relationship. Arch Microbiol 164:16-23. https://doi.org/10.1007/BF02568729.

7. van Kessel MAHJ, Speth DR, Albertsen M, Nielsen PH, Op den Camp HJM, Kartal B, Jetten MSM, Lücker S. 2015. Complete nitrification by a single microorganism. Nature 528:555-559. https://doi.org/10.1038/nature16459.

8. Daims H, Lebedeva EV, Pjevac P, Han P, Herbold C, Albertsen M, Jehmlich N, Palatinszky M, Vierheilig J, Bulaev A, Kirkegaard RH, von Bergen M, Rattei T, Bendinger B, Nielsen PH, Wagner M. 2015. Complete nitrification by Nitrospira bacteria. Nature 528:504-509. https://doi.org/10.1038/nature16461.

9. Bayer B, Saito MA, Mcllvin MR, Lücker S, Moran DM, Lankiewicz TS, Dupont CL, Santoro AE. 2021. Metabolic versatility of the nitrite-oxidizing bacterium Nitrospira marina and its proteomic response to oxygen-limited conditions. ISME J 15:1025-1039. https://doi.org/10.1038/s41396-020-00828-3.

10. Gruber-Dorninger C, Pester M, Kitzinger K, Savio DF, Loy A, Rattei T, Wagner M, Daims H. 2015. Functionally relevant diversity of closely related Nitrospira in activated sludge. ISME J 9:643-655. https://doi.org/ 10.1038/ismej.2014.156.

11. Orth JD, Thiele I, Palsson B $\emptyset$. 2010. What is flux balance analysis? Nat Biotechnol 28:245-248. https://doi.org/10.1038/nbt.1614.

12. Jang C, Chen L, Rabinowitz JD. 2018. Metabolomics and isotope tracing. Cell 173:822-837. https://doi.org/10.1016/j.cell.2018.03.055.

13. Mundinger $A B$, Lawson $C E$, Jetten MSM, Koch H, Lücker S. 2019. Cultivation and transcriptional analysis of a canonical Nitrospira under stable growth conditions. Front Microbiol 10:1325. https://doi.org/10.3389/ fmicb.2019.01325.

14. Lawson $C E$, Mundinger $A B$, Koch $H$, Jacobson TB, Weathersby $C A$, Jetten MSM, Pabst M, Amador-Noguez D, Noguera DR, McMahon K, Lücker S. 22 February 2021. Investigating the chemolithoautotrophic and formate metabolism of Nitrospira moscoviensis by constraint-based metabolic modeling and ${ }^{13} \mathrm{C}$-tracer analysis. BioRxiv https://doi.org/10.1101/2021.02.18 .431926 .

15. Caspi R, Billington $R$, Fulcher $C A$, Keseler IM, Kothari $A$, Krummenacker $M$, Latendresse M, Midford PE, Ong Q, Ong WK, Paley S, Subhraveti P, Karp PD. 2018. The MetaCyc database of metabolic pathways and enzymes. Nucleic Acids Res 46:D633-D639. https://doi.org/10.1093/nar/gkx935.

16. Arkin AP, Cottingham RW, Henry CS, Harris NL, Stevens RL, Maslov $S$, Dehal P, Ware D, Perez F, Canon S, Sneddon MW, Henderson ML, Riehl WJ, Murphy-Olson D, Chan SY, Kamimura RT, Kumari S, Drake MM, Brettin TS, Glass EM, Chivian D, Gunter D, Weston DJ, Allen BH, Baumohl J, Best AA, Bowen B, Brenner SE, Bun CC, Chandonia J-M, Chia J-M, Colasanti R, Conrad N, Davis JJ, Davison BH, DeJongh M, Devoid S, Dietrich E, Dubchak I, Edirisinghe JN, Fang G, Faria JP, Frybarger PM, Gerlach W, Gerstein M, Greiner A, Gurtowski J, Haun HL, He F, Jain R, Joachimiak MP, Keegan KP, Kondo S, et al. 2018. KBase: the United States Department of Energy systems biology knowledgebase. Nat Biotechnol 36:566-569. https://doi.org/10.1038/nbt.4163.

17. Bar-Even A, Noor E, Flamholz A, Milo R. 2013. Design and analysis of metabolic pathways supporting formatotrophic growth for electricity-dependent cultivation of microbes. Biochim Biophys Acta 1827:1039-1047. https://doi .org/10.1016/j.bbabio.2012.10.013.

18. Chadwick GL, Hemp J, Fischer WW, Orphan VJ. 2018. Convergent evolution of unusual complex I homologs with increased proton pumping capacity: energetic and ecological implications. ISME J 12:2668-2680. https://doi.org/ 10.1038/s41396-018-0210-1.

19. Feist AM, Palsson BO. 2010. The biomass objective function. Curr Opin Microbiol 13:344-349. https://doi.org/10.1016/j.mib.2010.03.003.

20. Lipski A, Spieck E, Makolla A, Altendorf K. 2001. Fatty acid profiles of nitrite-oxidizing bacteria reflect their phylogenetic heterogeneity. Syst Appl Microbiol 24:377-384. https://doi.org/10.1078/0723-2020-00049.
21. Bar-Even A, Flamholz A, Noor E, Milo R. 2012. Thermodynamic constraints shape the structure of carbon fixation pathways. Biochim Biophys Acta 1817:1646-1659. https://doi.org/10.1016/j.bbabio.2012.05.002.

22. Sumegi B, Sherry AD, Malloy CR. 1990. Channeling of TCA cycle intermediates in cultured Saccharomyces cerevisiae. Biochemistry 29:9106-9110. https://doi.org/10.1021/bi00491a002.

23. Wu F, Minteer S. 2015. Krebs cycle metabolon: structural evidence of substrate channeling revealed by cross-linking and mass spectrometry. Angew Chem Int Ed Engl 54:1851-1854. https://doi.org/10.1002/anie.201409336.

24. Zhang Y, Beard KFM, Swart C, Bergmann S, Krahnert I, Nikoloski Z, Graf A, Ratcliffe RG, Sweetlove LJ, Fernie AR, Obata T. 2017. Protein-protein interactions and metabolite channelling in the plant tricarboxylic acid cycle. Nat Commun 8:15212. https://doi.org/10.1038/ncomms15212.

25. Van Gool A, Laudelout H. 1966. Formate utilization by Nitrobacter wibogradskyi. Biochim Biophys Acta 127:295-301. https://doi.org/10.1016/0304 $-4165(66) 90384-9$.

26. O'Kelley JC, Nason A. 1970. Particulate formate oxidase from Nitrobacter agilis. Biochim Biophys Acta 205:426-436. https://doi.org/10.1016/0005 -2728(70)90108-8.

27. Sánchez-Andrea I, Guedes IA, Hornung B, Boeren S, Lawson CE, Sousa DZ, Bar-Even A, Claassens NJ, Stams AJM. 2020. The reductive glycine pathway allows autotrophic growth of Desulfovibrio desulfuricans. Nat Commun 11:5090. https://doi.org/10.1038/s41467-020-18906-7.

28. Stolyar S, Van Dien S, Hillesland KL, Pinel N, Lie TJ, Leigh JA, Stahl DA. 2007. Metabolic modeling of a mutualistic microbial community. Mol Syst Biol 3:92. https://doi.org/10.1038/msb4100131.

29. Zhuang K, Izallalen M, Mouser P, Richter H, Risso C, Mahadevan R, Lovley DR. 2011. Genome-scale dynamic modeling of the competition between Rhodoferax and Geobacter in anoxic subsurface environments. ISME J 5: 305-316. https://doi.org/10.1038/ismej.2010.117.

30. Mellbye BL, Giguere AT, Murthy GS, Bottomley PJ, Sayavedra-Soto LA, Chaplen FWR. 2018. Genome-scale, constraint-based modeling of nitrogen oxide fluxes during coculture of Nitrosomonas europaea and Nitrobacter winogradskyi. mSystems 3:e00170-17. https://doi.org/10.1128/ mSystems.00170-17.

31. Off S, Alawi M, Spieck E. 2010. Enrichment and physiological characterization of a novel Nitrospira-like bacterium obtained from a marine sponge. Appl Environ Microbiol 76:4640-4646. https://doi.org/10.1128/AEM.00320-10.

32. Nowka B, Daims H, Spieck E. 2015. Comparison of oxidation kinetics of nitriteoxidizing bacteria: nitrite availability as a key factor in niche differentiation. Appl Environ Microbiol 81:745-753. https://doi.org/10.1128/AEM.02734-14.

33. Meyer P, Cecchi G, Stolovitzky G. 2014. Spatial localization of the first and last enzymes effectively connects active metabolic pathways in bacteria. BMC Syst Biol 8:131. https://doi.org/10.1186/s12918-014-0131-1.

34. Williams TCR, Sweetlove LJ, Ratcliffe RG. 2011. Capturing metabolite channeling in metabolic flux phenotypes. Plant Physiol 157:981-984. https://doi.org/10.1104/pp.111.184887.

35. Sweetlove L, Fernie AR. 2018. The role of dynamic enzyme assemblies and substrate channelling in metabolic regulation. Nat Commun 9:2136. https://doi.org/10.1038/s41467-018-04543-8.

36. Bulutoglu B, Garcia KE, Wu F, Minteer SD, Banta S. 2016. Direct evidence for metabolon formation and substrate channeling in recombinant TCA cycle enzymes. ACS Chem Biol 11:2847-2853. https://doi.org/10.1021/ acschembio.6b00523.

37. Spieck E, Lipski A. 2011. Cultivation, growth physiology, and chemotaxonomy of nitrite-oxidizing bacteria. Methods Enzymol 486:109-130. https:// doi.org/10.1016/B978-0-12-381294-0.00005-5.

38. Henry CS, DeJongh M, Best AA, Frybarger PM, Linsay B, Stevens RL. 2010. High-throughput generation, optimization and analysis of genome-scale metabolic models. Nat Biotechnol 28:977-982. https://doi .org/10.1038/nbt.1672.

39. Varma A, Palsson BO. 1994. Stoichiometric flux balance models quantitatively predict growth and metabolic by-product secretion in wild-type Escherichia coli W3110. Appl Environ Microbiol 60:3724-3731. https://doi .org/10.1128/aem.60.10.3724-3731.1994.

40. Ebrahim A, Lerman JA, Palsson BØ, Hyduke DR. 2013. COBRApy: COnstraints-Based Reconstruction and Analysis for Python. BMC Syst Biol 7: 74. https://doi.org/10.1186/1752-0509-7-74.

41. Carnicer M, Baumann K, Töplitz I, Sánchez-Ferrando F, Mattanovich D, Ferrer P, Albiol J. 2009. Macromolecular and elemental composition analysis and extracellular metabolite balances of Pichia pastoris growing at different oxygen levels. Microb Cell Fact 8:65. https://doi.org/10 $.1186 / 1475-2859-8-65$. 
42. Herbert D, Phipps PJ, Strange RE. 1971. Chemical Analysis of Microbial Cells, p 209-344. In Norris JR, Ribbons DW (ed), Methods in microbiology. Academic Press, Cambridge, MA.

43. Izard J, Limberger RJ. 2003. Rapid screening method for quantitation of bacterial cell lipids from whole cells. J Microbiol Methods 55:411-418. https:// doi.org/10.1016/S0167-7012(03)00193-3.

44. Benthin S, Nielsen J, Villadsen J. 1991. A simple and reliable method for the determination of cellular RNA content. Biotechnol Tech 5:39-42. https://doi.org/10.1007/BF00152753.

45. Feist AM, Henry CS, Reed JL, Krummenacker M, Joyce AR, Karp PD, Broadbelt LJ, Hatzimanikatis V, Palsson BØ. 2007. A genome-scale metabolic reconstruction for Escherichia coli K-12 MG1655 that accounts for 1260 ORFs and thermodynamic information. Mol Syst Biol 3:121. https:// doi.org/10.1038/msb4100155.

46. Su X, Lu W, Rabinowitz JD. 2017. Metabolite spectral accuracy on Orbitraps. Anal Chem 89:5940-5948. https://doi.org/10.1021/acs.analchem.7b00396.

47. Åberg J, Wallin B. 2014. Evaluating a fast headspace method for measuring DIC and subsequent calculation of $\mathrm{pCO}_{2}$ in freshwater systems. Inland Waters 4:157-166. https://doi.org/10.5268/IW-4.2.694.
48. Köcher T, Pichler P, Swart R, Mechtler K. 2012. Analysis of protein mixtures from whole-cell extracts by single-run nanoLC-MS/MS using ultralong gradients. Nat Protoc 7:882-890. https://doi.org/10.1038/nprot.2012.036.

49. Käll L, Krogh A, Sonnhammer ELL. 2004. A combined transmembrane topology and signal peptide prediction method. J Mol Biol 338:1027-1036. https://doi.org/10.1016/j.jmb.2004.03.016.

50. Liao Y, Smyth GK, Shi W. 2014. FeatureCounts: an efficient general purpose program for assigning sequence reads to genomic features. Bioinformatics 30:923-930. https://doi.org/10.1093/bioinformatics/btt656.

51. Krzywinski M, Schein J, Birol I, Connors J, Gascoyne R, Horsman D, Jones SJ, Marra MA. 2009. Circos: an information aesthetic for comparative genomics. Genome Res 19:1639-1645. https://doi.org/10.1101/gr.092759.109.

52. Vizcaíno JA, Côté RG, Csordas A, Dianes JA, Fabregat A, Foster JM, Griss J, Alpi E, Birim M, Contell J, O'Kelly G, Schoenegger A, Ovelleiro D, PérezRiverol Y, Reisinger F, Ríos D, Wang R, Hermjakob H. 2013. The Proteomics Identifications (PRIDE) database and associated tools: status in 2013. Nucleic Acids Res 41:D1063-D1069. https://doi.org/10.1093/nar/gks1262.

53. Neidhardt FC, Ingraham JL, Schaechter M. 1990. Physiology of the bacterial cell: a molecular approach. Sinauer Associates, Sunderland, MA. 